\title{
CLASH-VLT: Substructure in the galaxy cluster MACS J1206.2-0847 from kinematics of galaxy populations ${ }^{\star}, \star \star$
}

\author{
M. Girardi ${ }^{1,2}$, A. Mercurio ${ }^{3}$, I. Balestra ${ }^{2}$, M. Nonino ${ }^{2}$, A. Biviano ${ }^{2}$, C. Grillo ${ }^{4}$, P. Rosati ${ }^{5}$, M. Annunziatella ${ }^{1,2}$, \\ R. Demarco ${ }^{6}$, A. Fritz ${ }^{7}$, R. Gobat ${ }^{8}$, D. Lemze ${ }^{9}$, V. Presotto ${ }^{1}$, M. Scodeggio ${ }^{7}$, P. Tozzi ${ }^{10}$, G. Bartosch Caminha ${ }^{5}$, \\ M. Brescia ${ }^{3}$, D. Coe ${ }^{11}$, D. Kelson ${ }^{12}$, A. Koekemoer ${ }^{11}$, M. Lombardi ${ }^{13}$, E. Medezinski ${ }^{9}$, M. Postman ${ }^{11}$, \\ B. Sartoris ${ }^{1,2}$, K. Umetsu ${ }^{14}$, A. Zitrin ${ }^{15, \star \star \star}$, W. Boschin ${ }^{16,17,18}$, O. Czoske ${ }^{19}$, G. De Lucia ${ }^{2}$, U. Kuchner ${ }^{19}$, C. Maier ${ }^{19}$, \\ M. Meneghetti ${ }^{20,21,22}$, P. Monaco ${ }^{1,2}$, A. Monna ${ }^{23}$, E. Munari ${ }^{1,2}$, S. Seitz ${ }^{23,24}$, M. Verdugo ${ }^{19}$, and B. Ziegler ${ }^{19}$ \\ (Affiliations can be found after the references)
}

Received 30 December 2014 / Accepted 16 March 2015

\begin{abstract}
Aims. In the effort to understand the link between the structure of galaxy clusters and their galaxy populations, we focus on MACS J1206.2-0847 at $z \sim 0.44$ and probe its substructure in the projected phase space through the spectrophotometric properties of a large number of galaxies from the CLASH-VLT survey.

Methods. Our analysis is mainly based on an extensive spectroscopic dataset of 445 member galaxies, mostly acquired with VIMOS at VLT as part of our ESO Large Programme, sampling the cluster out to a radius $\sim 2 R_{200}\left(4 h_{70}^{-1} \mathrm{Mpc}\right.$ ). We classify 412 galaxies as passive, with strong $\mathrm{H} \delta$ absorption (red and blue galaxies), and with emission lines from weak to very strong. A number of tests for substructure detection are applied to analyze the galaxy distribution in the velocity space, in $2 \mathrm{D}$ space, and in $3 \mathrm{D}$ projected phase-space.

Results. Studied in its entirety, the cluster appears as a large-scale relaxed system with a few secondary, minor overdensities in 2D distribution. We detect no velocity gradients or evidence of deviations in local mean velocities. The main feature is the WNW-ESE elongation. The analysis of galaxy populations per spectral class highlights a more complex scenario. The passive galaxies and red strong $\mathrm{H} \delta$ galaxies trace the cluster center and the WNW-ESE elongated structure. The red strong $\mathrm{H} \delta$ galaxies also mark a secondary, dense peak $\sim 2 h_{70}^{-1} \mathrm{Mpc}$ at ESE. The emission line galaxies cluster in several loose structures, mostly outside $R_{200}$. Two of these structures are also detected through our 3D analysis. The observational scenario agrees with MACS J1206.2-0847 having WNW-ESE as the direction of the main cluster accretion, traced by passive galaxies and red strong $\mathrm{H} \delta$ galaxies. The red strong $\mathrm{H} \delta$ galaxies, interpreted as poststarburst galaxies, date a likely important event $1-2$ Gyr before the epoch of observation. The emission line galaxies trace a secondary, ongoing infall where groups are accreted along several directions.
\end{abstract}

Key words. galaxies: clusters: individual: MACS J1206.2-0847 - galaxies: clusters: general - galaxies: kinematics and dynamics galaxies: evolution - cosmology: observations

\section{Introduction}

In the hierarchical scenario for large-scale structure formation, clusters of galaxies are not relaxed structures. Numerical simulations show that clusters form preferentially through anisotropic accretion of subclusters along the large-scale structure (LSS) filaments (Colberg et al. 1998, 1999 and references therein). From the observational point of view, it is well known that a large fraction of clusters $(30 \%-70 \%)$ contain substructures, as shown by studies based on optical (e.g., Baier \& Ziener 1977; Geller \& Beers 1982; Girardi et al. 1997; Ramella et al. 2007; Wen \& Han 2013), on X-ray data (e.g., Jones \& Forman 1999; Buote 2002; Jeltema et al. 2005; Zhang et al. 2009; Böhringer et al. 2010), and on gravitational lensing techniques (e.g., Athreya et al. 2002; Dahle et al. 2002; Smith et al. 2005: Grillo et al. 2014a), indicating that past signatures of cluster accretion are quite common. Much progress has been made in the study of cluster accretion phenomena (see Feretti et al. 2002, for a general

\footnotetext{
* Based in large part on data acquired at the ESO VLT (prog.ID 186.A-0798).

$\star \star$ Full Table 2 is only available at the CDS via anonymous ftp to cdsarc.u-strasbg.fr (130.79.128.5) or via

http: //cdsarc.u-strasbg.fr/viz-bin/qcat?]/A+A/579/A4

$\star \star \star$ Hubble Fellow.
}

review) and recent, dedicated studies have often focused on a few major, ongoing cluster mergers, for instance in the context of Dynamical Analysis Radio Clusters project (DARC, e.g., Girardi et al. 2011) and MUlti-Wavelength Sample of Interacting Clusters project (MUSIC, Maurogordato et al. 2011). Other dedicated studies have focused on larger scales, i.e., on cluster accretion through filaments (e.g., Fadda et al. 2008; Dietrich et al. 2012). On-going cluster formation is also evident in distant clusters as pioneering studies have shown that most clusters identified at $z \geq 0.8$ are elongated, clumpy, or with a filamentary structure (e.g., Donahue et al. 1998; Gioia et al. 1999; Demarco et al. 2007; Fassbender et al. 2011).

It is well established that the properties of cluster galaxies differ from those of field galaxies and that clusters are characterized by radial gradients. Galaxies in denser, central regions are usually of earlier morphological type, redder color, and lower star formation rate (SFR; e.g., Gerken et al. 2004). However, the precise details of the connection between galaxy evolution and cluster environment are still unknown. Several physical mechanisms can be involved in modifying galaxies in the cluster environment (see Fig. 10 by Treu et al. 2003, and refs. therein). Moreover, it is clear that a scenario in which galaxies are accreted in a static cluster environment is too simplistic. In fact, there is evidence of a connection between the fraction of 
active galaxies and the presence and/or the amount of substructure, generally interpreted as an enhanced star formation (SF) in member galaxies caused by the process of cluster mergers (e.g., Caldwell \& Rose 1997; Ferrari et al. 2005; Owen et al. 2005). An alternative interpretation is that quenching SF is less effective in just forming multicomponent clusters (see Cohen et al. 2014 for further discussions). From a theoretical point of view, during a major cluster merger, the SF in gas-rich galaxies can decrease or, alternatively, be triggered depending on the specific interaction between the intracluster medium (ICM) and the interstellar gas within the galaxies (e.g., Fujita et al. 1999; Bekki et al. 2010). Time-dependent tidal fields of merging groups and clusters of galaxies can also trigger secondary starbursts in their member galaxies (Bekki 1999).

In this context, poststarburst galaxies (PSBs, Dressler \& Gunn 1983) play a crucial role. In fact, their spectral signature, i.e., strong Balmer absorption lines and weak or no emission lines, is generally attributed to a vigorous SF that has recently decreased significantly or ceased altogether. More properly, one can think of a real poststarburst phase or, alternatively, a starforming truncated phase (e.g., Dressler et al. 2009). The last SF event in PSBs galaxies ended a few Myr to 2 Gyr before the time of observation, on the basis of the lifetime of the stars responsible for the strong Balmer lines (e.g., Poggianti \& Barbaro 1996, 1997; Balogh et al. 1999; Poggianti et al. 1999; Mercurio et al. 2004, hereafter M04). More specifically, the typical time elapsed since the last SF in the red PSBs lies in the range 1-2 Gyr, while in the blue PSBs the interruption of SF occurred within the last 0.5 Gyr (M04). The location of PSBs in the parent cluster can give important information on the driving physical mechanism. For instance, the synchronized triggered SF in the cluster merging simulations of Bekki et al. (2010) gives origin to a population of PSBs having a spatial distribution different from the rest of the galaxy population. Analyzing nine clusters at $z \sim 1$ drawn from the GCLASS survey, Muzzin et al. (2014) have found that PSBs appear to trace a coherent ring in the projected phase space. Alternatively, Dressler et al. (2013) have found that in the IMACS Cluster Building Survey (five rich clusters at $0.31<z<0.55$ ) the PSB population traces the same spatial distribution of the passive population.

Recently, significant interest has focused on cluster outskirts, too. In fact, although there is evidence that SF is suppressed on average outside $2 R_{200}{ }^{1}$ in both low- and high-redshift clusters (e.g., Lewis et al. 2002; Gomez et al. 2003; Rasmussen et al. 2012), several studies have associated starburst features with infalling groups (e.g., Owen et al. 2005; M04; Poggianti et al. 2004; Oemler et al. 2009). In nearby clusters an increase of SF along the filaments feeding the clusters has been found (e.g., Fadda et al. 2008). This seems particularly true around $1.5-2 R_{200}$ and for galaxies belonging to groups, suggesting that a relatively high density in the infalling regions promotes interactions between galaxies (e.g., galaxy-galaxy harassment) and following momentary bursts of SF (Scott et al. 2007; Mahajan et al. 2012). Recent simulations suggest that cluster galaxies may be pre-processed before the infall into the cluster environment, although the postprocessing is important as well (Vijayaraghavan \& Ricker 2013).

Optical multiband data and particularly multiobject spectroscopy are a consolidated way to investigate cluster substructure and cluster merging phenomena (e.g., Girardi \& Biviano 2002). The few recent studies of clusters with hundreds of

\footnotetext{
1 The radius $R_{\delta}$ is the radius of a sphere with mass overdensity $\delta$ times the critical density at the redshift of the galaxy system.
}

spectroscopic members have shown the power of using large spectroscopic catalogs to study cluster internal structure (e.g., Owers et al. 2011; Munari et al. 2014). In some studies, the correlation of passive and active galaxies populations with the substructure of the parent cluster has been investigated (e.g., Czoske et al. 2002; M04; Mercurio et al. 2008; Oemler et al. 2009; Ma et al. 2010). Very large spectroscopic datasets are needed to study cluster substructure in phase space for different spectral types and to infer cluster assembly history. The present study represents a pilot study in this new direction. It focuses on the galaxy cluster MACS J1206.2-0847 (hereafter MACS1206; Ebeling et al. 2009) at $z \sim 0.44$, discovered and first described in the REFLEX Galaxy Cluster Survey catalog (RXC J1206.20848; Böhringer et al. 2004).

\subsection{MACS1206 and the CLASH-VLT programme}

The subject of this study, the galaxy cluster MACS1206 is part of the survey of 25 target clusters of the HST MultiCycle Treasury program "Cluster Lensing And Supernova" (CLASH, P.I.: M. Postman; Postman et al. 2012). Our study is based on an extensive spectroscopic dataset collected within the ESO Large Programme 186.A-0798 "Dark Matter Mass Distributions of Hubble Treasury Clusters and the Foundations of $\Lambda$ CDM Structure Formation Models" (hereafter CLASHVLT, P.I.: P. Rosati; Rosati et al. 2014). This program aims at obtaining spectra for at least 500 cluster members for each of the 14 southern CLASH clusters with $0.2<z<0.6$, using the VIsible Multi-Object Spectrograph (VIMOS) at VLT (CLASHVLT survey). The study of the combined evolution of galaxies and parent cluster requires i) the spectroscopic identification of a large number of cluster galaxies in each cluster; ii) sampling a region out to at least twice $R_{200}$; and iii) a range of cluster redshifts covering a relevant epoch cluster formation. Specifically, the past 5 Gyr play a crucial role in cluster assembly history since the overall morphological content, the fraction of galaxies of different spectral types, and the SF history of cluster galaxies are known to significantly evolve since $z \sim 0.5$ (e.g., Butcher $\&$ Oemler 1984; Couch \& Sharples 1987; Dressler et al. 1997; Fritz et al. 2005; Poggianti et al. 2009). Useful complementary information from optical, X-ray and radio data, and high-resolution HST imaging is available, too. Other CLASH-based studies, focused on other aspects of MACS1206, have already been presented and are referred to and discussed throughout this paper (Umetsu et al. 2012; Zitrin et al. 2012; Biviano et al. 2013, B13; Annunziatella et al. 2014; Grillo et al. 2014b; Presotto et al. 2014).

The paper is organized as follows. We present our catalog and galaxy spectral type classification in Sects. 2 and 3, respectively. Section 4 concentrates on the analysis of cluster structure. Section 5 is devoted to the interpretation and discussion of our results. We give our summary and conclusions in Sect. 6 .

Unless otherwise stated, we give errors at the $68 \%$ confidence level (hereafter c.l.). Throughout this paper, we use $H_{0}=70 \mathrm{~km} \mathrm{~s}^{-1} \mathrm{Mpc}^{-1}$ in a flat cosmology with $\Omega_{0}=0.3$ and $\Omega_{\Lambda}=0.7$. In the adopted cosmology, $1^{\prime}$ corresponds to $0.341 h_{70}^{-1} \mathrm{Mpc}$ at the cluster redshift.

\section{Redshift catalog and member selection}

The full redshift catalog of MACS1206 consists of 3292 objects, with measured redshifts mostly acquired as part of our ESO Large Programme 186.A-0798 (P.I.: Piero Rosati) using 
the VIMOS at VLT/UT3. Additional archival VIMOS data have been homogeneously reduced from programs 169.A-0595 (P.I.: Hans Böhringer) and 082.A-0922 (P.I. Mike Lerchster) and have provided 952 spectra in the cluster field. All data are reduced with the same VIPGI package (see Scodeggio et al. 2005). Four redshift quality classes were defined "secure" $(\mathrm{QF}=3)$, "based on a single-emission-line" $(\mathrm{QF}=9)$, "likely" $(\mathrm{QF}=2)$, and "insecure" (QF = 1), having $>99.99 \%, \sim 92 \%, \sim 75 \%$, and $<40 \%$ probability to be correct, respectively. Further details on the ESO Large Programme 186.A-0798 are reported elsewhere (Rosati et al. 2014 and in Rosati et al., in prep.). The catalog also includes 22 spectra acquired with FORS2 at VLT as part of ESO Programme ID 089.A-0879 (P.I.: Raphael Gobat) and additional literature redshifts taken from Lamareille et al. (2006, 1 object), Jones et al. (2004, 3 objects), Ebeling et al. (2009, 25 objects), and 21 objects observed with IMACS-GISMO at the Magellan telescope (Daniel Kelson private communication). The full catalog, with the exception of $\mathrm{QF}=1$ redshifts has already been used by B13 and Lemze et al. (2013) and is publicly available. In this paper, we do not consider redshifts with $\mathrm{QF}=1$ and 2. Our reference sample includes 1920 galaxies.

Biviano et al. (2013) quantified the completeness of the spectroscopic catalog by computing the ratio between the $R_{\mathrm{C}}$-band number counts of objects with measured redshift and all photometric objects. In particular, B13 show that in the virial region the completeness is $\sim 0.6$ at $R_{\mathrm{C}} \sim 20, \sim 0.3$ at $R_{\mathrm{C}} \sim 23$, and then declines very sharply (see their Fig. 4). However, although the redshift catalog covers a fraction of the photometric objects, it is essentially unbiased with respect to galaxy color. Biviano et al. (2013) also evaluated the spatial completeness of the spectroscopic sample finding a good uniformity with a mild radiallydependent incompleteness. The completeness varies with radius from 0.6 at the cluster center to 0.2 at the external cluster limit (see their Fig. 5). Only two low-overdensity features are shown in their Fig. 5: the first in the center, elongated towards the south direction, the second $10^{\prime}$ to the east. Our sample and that of B13 do not differ in their spatial distributions according to the 2D Kolmogorov-Smirnov test (hereafter 2DKS-test, Fasano \& Franceschini 1987). A posteriori, we verified that none of the galaxy clumps or subclusters we discuss in this paper resemble the specific overdensity features detected in the completeness map by B13 (cf. their Fig. 5 with our results and figures in Sect. 3.2). We conclude that our results are not affected by the redshift spatial incompleteness.

To select cluster members, we applied the two-step method called "peak+gap" $(\mathrm{P}+\mathrm{G})$ already applied by B13 and previous studies (e.g., Girardi et al. 2011 and refs. therein). The method is a combination of the 1D adaptive-kernel method DEDICA (Pisani 1993) and the "shifting gapper", which uses both position and velocity information (see Fadda et al. 1996; Girardi et al. 1996). For the center of MACS1206, we adopted the position of the brightest cluster galaxy $\left(\mathrm{BCG}, \mathrm{RA}=12^{\mathrm{h}} 06^{\mathrm{m}} 12^{\mathrm{s}} .15\right.$, Dec $\left.=-08^{\circ} 48^{\prime} 03.3^{\prime \prime}(\mathrm{J} 2000)\right)$. In the first step, we detected MACS1206 as a peak at $z \sim 0.4385$ populated by 466 galaxies. The second step leads to 445 cluster members.

By applying the biweight estimator to the 445 cluster members (Beers et al. 1990, ROSTAT software), we computed a mean cluster line-of-sight (LOS) velocity $\langle V\rangle=\langle c z\rangle=(131843 \pm$ 49) $\mathrm{km} \mathrm{s}^{-1}$, corresponding to a mean cluster redshift $\langle z\rangle=$ $0.4398 \pm 0.0002$. We estimated the LOS velocity dispersion, $\sigma_{V}$, by using the biweight estimator and applying the cosmological correction and the standard correction for velocity errors (Danese et al. 1980). We obtained $\sigma_{V}=1035_{-45}^{+27} \mathrm{~km} \mathrm{~s}^{-1}$, where errors are estimated through a bootstrap technique. Both $\langle V\rangle$ and
$\sigma_{V}$ are in agreement with the values obtained by B13 using a larger galaxy sample. Out of the reference sample of 445 cluster members (hereafter the TOT sample), we also considered the sample of 249 galaxies within $R_{200}$ (hereafter the R200 sample). The estimate of $R_{200}$ spans the range $1.96-2.08 h_{70}^{-1} \mathrm{Mpc}$, with a typical $1 \sigma$ uncertainty of $\sim 5 \%$, depending on the method of analysis (see Table 2 of B13). Hereafter the value $R_{200}=$ $2 h_{70}^{-1}$ Mpc is assumed.

Throughout the paper we also use color and morphological information coming from the Subaru Suprime-Cam data. These data were retrieved from the SMOKA archive ${ }^{2}$ and reduced following the same steps as adopted by Nonino et al. (2009). Further information can be found in Presotto et al. (2014) and in Mercurio et al. (in prep.), where this photometric information is more extensively used.

\section{Spectral type classification and galaxy populations}

\subsection{Spectral type classification}

Out of a total of 445 member galaxies, we have the spectra of 415 galaxies acquired at VIMOS at VLT (405 objects) and FORS2 at VLT (10 objects), other redshifts coming from the literature or other sources (see Sect. 2). Following most of the literature, we used emission lines and $\mathrm{H} \delta$ absorption lines to classify galaxies. We used wavelength-calibrated and flux-calibrated galaxy spectra as obtained from VIPGI (Scodeggio et al. 2005). We corrected for galactic extinction following Schlegel et al. (1998) and using the IRAF $^{3}$ task DEREDDEN included in the package ONEDSPEC. Finally, the calibrated and dereddened spectra were corrected for the measured velocity dispersion by using DISPCOR.

We measured the equivalent widths (EWs) for the emission lines [OII], [OIII], and when available $\mathrm{H} \alpha$. All the spectra cover the [OII] region with the exception of 18 galaxies. We also measured the EW for $\mathrm{H} \delta$ (see M04 for the definition of wavelength ranges). We detected 185 galaxies with evidence of emission lines. The 185 galaxies with emission lines were divided in four classes depending on the strength of the [OII] emission line, or of the [OIII] emission line for the four cases where the [OII] region is not covered. In these four cases we assumed a $[\mathrm{OIII}] /[\mathrm{OII}]$ flux ratio equal to one. We considered the wELG, mELG, sELG, and vsELG classes, i.e., those formed of galaxies with weak, medium, strong, and very strong emission lines (see Table 1). Figure 1 shows the co-added spectra for emission line galaxies and other classes. All the emission line galaxies, with the exception of wELGs, show similar distributions of the clustercentric distances (see Sect. 3.2), and we aggregated them in the ELG class.

Among the non-emission line galaxies, the 44 galaxies with $E W(\mathrm{H} \delta)>3 \AA$ were classified as strong $\mathrm{H} \delta$ absorption galaxies (HDS sample), interpreted as PSBs or galaxies with truncated $\mathrm{SF}$ (see Sect. 1). Compared to red galaxies, blue galaxies require larger $E W(\mathrm{H} \delta)$ to be identified as galaxies that have a peculiar star-forming activity. Thus, a more precise way to classify strong $\mathrm{H} \delta$ galaxies uses the diagram of $\mathrm{EW}(\mathrm{H} \delta)$ vs. $(B-R)$ color or,

\footnotetext{
2 http://smoka.nao.ac.jp/SUPsearch

3 IRAF is distributed by the National Optical Astronomy Observatories, which are operated by the Association of Universities for Research in Astronomy, Inc., under cooperative agreement with the National Science Foundation.
} 
Table 1. Spectral type classification.

\begin{tabular}{llrcc}
\hline \hline Galaxy characteristics & Class & $N_{\mathrm{g}}$ & $E W([\mathrm{OII}])^{a}$ & $E W(\mathrm{H} \delta)$ \\
\hline Passive & $\mathrm{PAS}$ & 183 & absent & absent \\
Strong H $\delta$ absorption \& red color & $\mathrm{HDSr}^{b}$ & 38 & absent & $>3 \AA$ \\
Strong H $\delta$ absorption \& blue color & $\mathrm{HDSb}^{b}$ & 5 & absent & $>5 \AA$ \\
Weak emission lines & $\mathrm{wELG}^{a}$ & 17 & $>-7 \AA$ & any \\
Medium emission lines & $\mathrm{mELG}^{a}$ & 24 & $(-15 \AA,-7 \AA]$ & any \\
Strong emission lines & $\mathrm{sELG}^{a}$ & 101 & $(-40 \AA,-15 \AA]$ & any \\
Very strong emission lines & $\mathrm{vsELG}^{a}$ & 43 & $\leq-40 \AA$ & any \\
\hline
\end{tabular}

Notes. ${ }^{(a)}$ In four cases EW([OIII]) were used, see the text and Table 2; ${ }^{(b)}$ The HDS class $\left(N_{\mathrm{g}}=44\right)$ is formed of HDSr and HDSb galaxies, and an additional galaxy with strong $\mathrm{H} \delta$ absorption, which can be classified as neither HDSr nor HDSb; ${ }^{(c)}$ The ELG class $\left(N_{\mathrm{g}}=168\right)$ is formed of mELG, sELG, and vsELG. Throughout the paper we use PASs, HDSrs, HDSbs, and w/m/s/vsELGs to refer to the respective member galaxies.

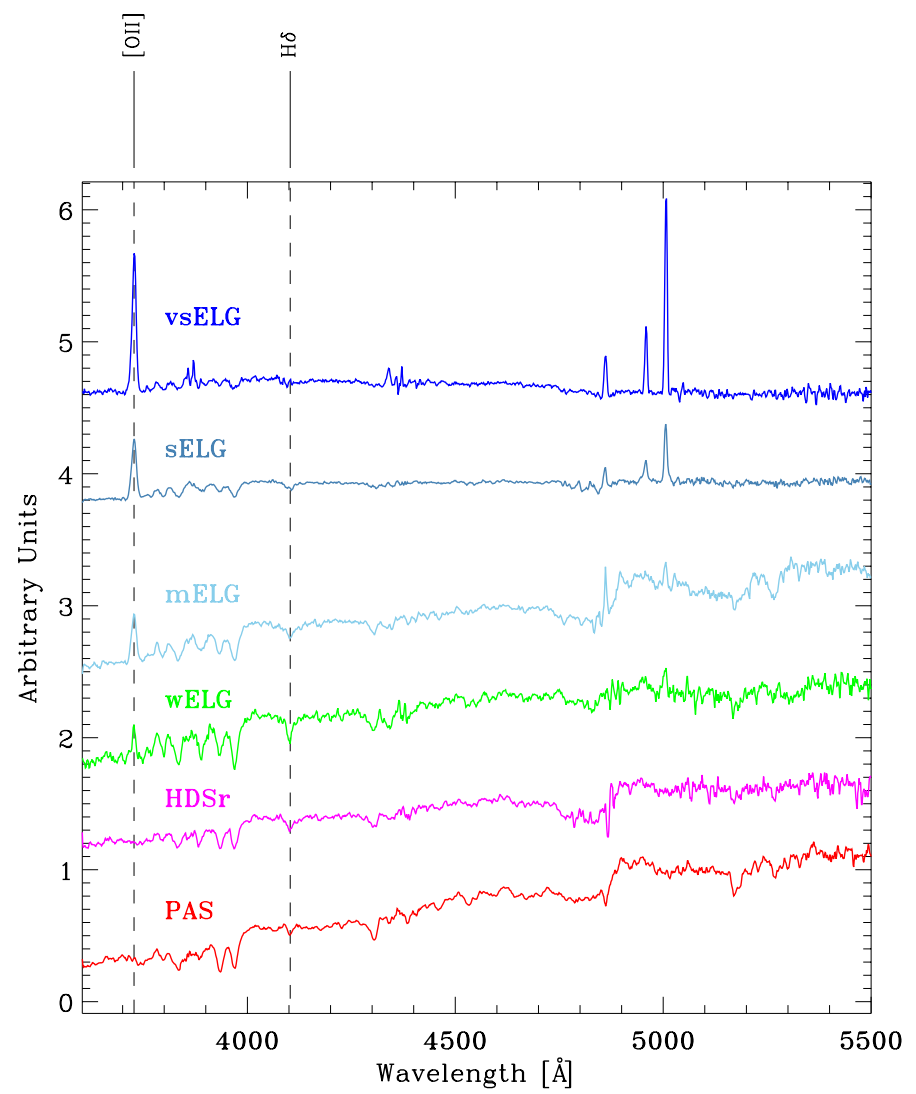

Fig. 1. Co-added (rest-frame) spectra of cluster galaxies per spectral class. The positions of $[\mathrm{OII}]$ and $\mathrm{H} \delta$ lines used in the classification are indicated.

alternatively, vs. the strength of the $4000 \AA$ break, which correlates with color (Couch \& Sharples 1987; Barger et al. 1996; Balogh et al. 1999). We considered 38 strong $\mathrm{H} \delta$ galaxies with red colors and $E W(\mathrm{H} \delta)>3 \AA$ ( $\mathrm{HDSr}$ ) and 5 strong $\mathrm{H} \delta$ galaxies with blue colors and $E W(\mathrm{H} \delta)>5 \AA$ (HDSb). In this separation, we followed M04 using their threshold in the differential color $\left(B-R_{\mathrm{C}}\right)_{\text {diff }}=-0.5$, where the differential color is defined as the observed color minus the color of the color-magnitude relation at the given magnitude. The color-magnitude relation was obtained as fitted to the spectroscopically confirmed members $\left(B-R_{\mathrm{C}}=3.587-0.0714 R_{\mathrm{C}}\right)$, where $R_{\mathrm{C}}$ indicates the Kron magnitudes and the $B-R_{\mathrm{C}}$ color is computed on aperture magnitudes (Mercurio et al., in prep.). In their original study, M04 based their HDS classification scheme directly on the strength of the
$4000 \AA$ A break, while we use the corresponding color. The colormagnitude correction allows us to take into account the large magnitude range spanned by our dataset.

With the exception of three galaxies whose signal-to-noise is too low according to our standards ( $S / N \sim 2$ for the $\mathrm{H} \delta$ absorption line), the remaining 183 galaxies were classified as passive (PAS). We note that the BCG is classified as a passive galaxy in spite of the presence of a [OII] emission line in the VIMOS spectrum since the HST photometric data suggest that this line can be associated with a blue compact source and/or peculiar features blended with the BCG (see Presotto et al. 2014 for the relevant discussion).

Summarizing, we have spectral type classifications for 412 galaxies, 217 galaxies within $R_{200}$. The spectroscopic catalog is electronically published in Table 2, available at CDS. Table 1 summarizes the numbers of galaxies assigned to each spectral class.

To compare our spectral-classification statistics to that of Dressler et al. (2013), which considers five rich clusters at $0.31<z<0.54$, we also computed the fractions per spectral class within $1.5 R_{200}$ and $R_{\mathrm{C}}<22.3$. We obtained the fraction $(\mathrm{PAS}+\mathrm{HDS}) / \mathrm{TOT}=67 \%$ and $\mathrm{HDS} / \mathrm{PAS}=24 \%$. These values do not change significantly when taking into account the radial spatial incompleteness (see Fig. 5 of B13 and our Sect. 2), i.e., normalizing the number of galaxies in different cluster radial bins $\left(R \leq 0.4 h_{70}^{-1} \mathrm{Mpc}, 0.4<R \leq 1 h_{70}^{-1} \mathrm{Mpc}, 1<R \leq 2 h_{70}^{-1} \mathrm{Mpc}\right.$, $\left.R>2 h_{70}^{-1} \mathrm{Mpc}\right)$ using different spatial completeness corrections (i.e., dividing by $0.6,0.5,0.4,0.3)$. Our values are in good agreement with the corresponding fractions (PAS+PSB)/TOT and PSB/PAS reported by Dressler et al. (2013, see the cluster values in their Fig. 16 and Table 4).

We also used some results from the morphological analysis of our galaxy sample as derived from Suprime-Cam at Subaru data (Mercurio et al., in prep.). The PAS, HDSr, wELG, and ELG sequences of spectral type populations correspond to bluer and bluer galaxies (see Fig. 2). For each spectral class with the exception of HDSb, Fig. 3 shows the distribution of Sérsic index $n$, as determined in the $R_{\mathrm{C}}$-band Subaru image using the GALAPAGOS software (Barden et al. 2012) and a single Sérsic profile. The value of $n$ is larger for more prominent bulges and $n=2-2.5$ can be considered the transition value between disk-dominated and bulge-dominated galaxies (e.g., M04; Barden et al. 2005; Fisher \& Drory 2008). We note a good correlation between the Sérsic index $n$ and spectral classes, with PASs mostly bulge-dominated and ELGs mostly disk-dominated, while HDSr and wELG galaxies have intermediate values of $n$. 
Table 2. Spectral classification of galaxies in the cluster MACS J1206.2-0847.

\begin{tabular}{cccccccc}
\hline \hline ID & $\alpha(\mathrm{J} 2000)$ & $\delta(\mathrm{J} 2000)$ & $z$ & $B-R_{\mathrm{C}}$ & $\begin{array}{c}E W([\mathrm{OII}]) \\
\AA\end{array}$ & $\begin{array}{c}E W(\mathrm{H} \delta) \\
\AA\end{array}$ & Class \\
& & & & & & $\AA$ \\
\hline 1 & $12: 06: 55.15$ & $-08: 56: 29.7$ & 0.4367 & $1.77 \pm 0.01$ & $-1.50 \pm 0.30$ & $6.90 \pm 0.20$ & wELG \\
2 & $12: 06: 05.16$ & $-08: 53: 20.6$ & 0.4360 & $1.26 \pm 0.01$ & $-2.80 \pm 0.50$ & $2.50 \pm 0.50$ & wELG \\
\hline
\end{tabular}

Notes. Column 1: running ID for galaxies in the presented sample; Cols. 2 and 3: RA and Dec (J2000); Col. 4: spectroscopic redshift, $z$; Col. 5 $B-R_{\mathrm{C}}$ color; Cols. 6, 7: $[\mathrm{OII}]$ and $\mathrm{H} \delta$ equivalent widths. We list "..." when the $\mathrm{EW}$ cannot be measured because the line does not lie within the available wavelength range. We list " 0.00 " for EW[OII] when no emission is observed; Col. 8: the spectral classification. This table is available at the CDS. A portion is shown here for guidance regarding its form and content.

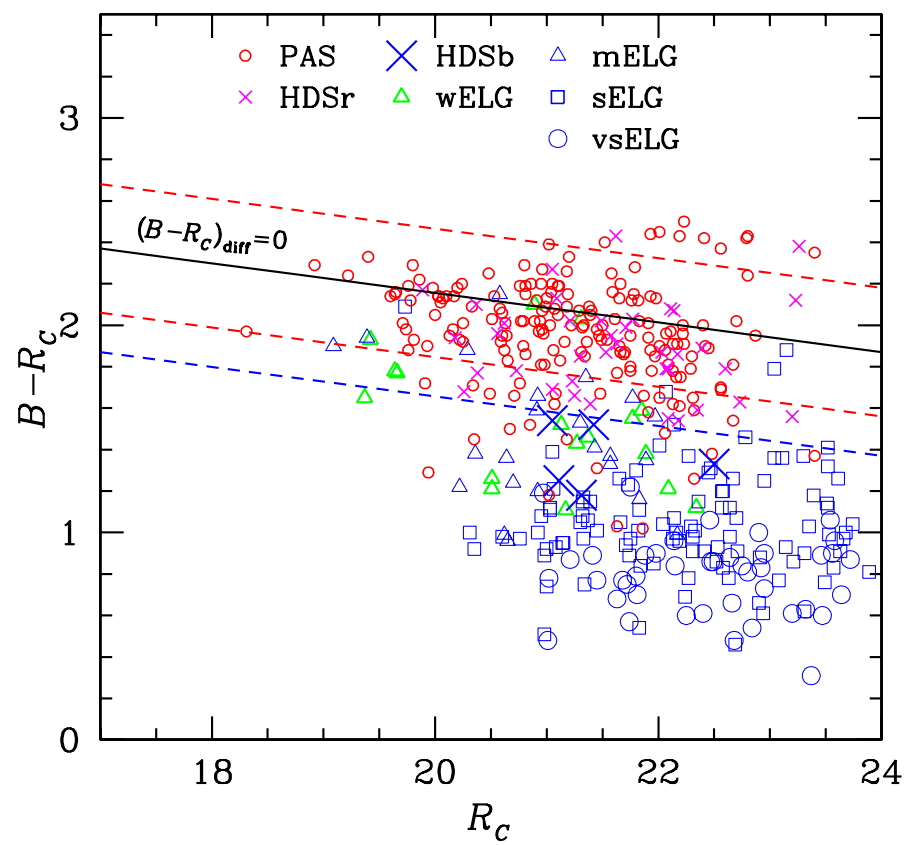

Fig. 2. $B-R_{\mathrm{C}}$ vs. $R_{\mathrm{C}}$ color-magnitude diagram with our seven spectral classes. The black solid line is the color-magnitude relation, $\left(B-R_{\mathrm{C}}\right)_{\mathrm{diff}}=0$. The blue dashed line indicates the $\left(B-R_{\mathrm{C}}\right)_{\mathrm{diff}}=-0.5$ value which we use to separate HDSr and HDSb following M04. The two red dashed lines indicate the locus of the red sequence galaxies, $\left|\left(B-R_{\mathrm{C}}\right)_{\text {diff }}\right|<0.3$.

\subsection{Space and velocity distributions of galaxy populations}

Figure 4 shows that PAS and HDSr galaxies are more spatially clustered than ELGs. To make a quantitative comparison among different galaxy populations, we applied the Kruskall-Wallis test to the clustercentric distances $R$ (KW-test, e.g., Ledermann 1982). This test is a non-parametric method for testing whether samples originate from the same distribution. The KW test leads to significant results ( $>99.99 \%$ c.l.); at least one of the samples is different from the other samples. In Fig. 5 there is a clear dichotomy between the population formed of PAS, HDSr, and wELG galaxies and the population formed of mELG, sELG, and vsELG galaxies, the galaxies of the former group being more clustered. We also compared spectral classes two by two. We applied the 1D Kolmogorov-Smirnov test (hereafter 1DKStest; e.g., Ledermann 1982) to compare the distributions of clustercentric distances. When comparing one of the PAS, HDSr, wELG classes with one of the mELG, sELG, vsELG classes we detected significant differences (at $>98 \%$ c.l.). The comparison between PAS and HDSr, PAS and wELG, and HDSr and wELG shows no differences, and similarly the comparison between mELG and sELG, mELG and vsELG, and sELG and vsELG.

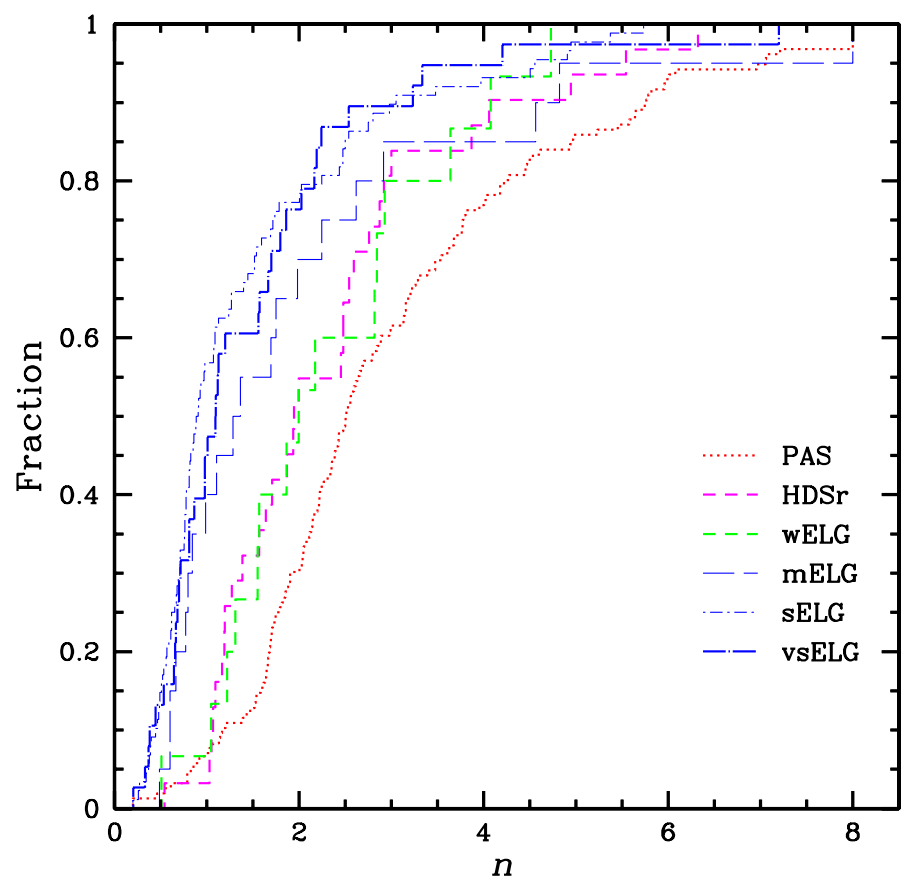

Fig. 3. Cumulative distribution of Sérsic index of galaxies per spectral class.

The results of the 1DKS-test confirm the dichotomy between the population formed of PAS, HDSr, and wELG galaxies and that formed of mELG, sELG and vsELG galaxies and prompted us to treat mELGs, sELGs, and vsELGs together in the combined ELG class, separately from wELGs. This is a more detailed view, across several spectral types, of the well-known spatial segregation of spiral/elliptical galaxies and of blue/red galaxies in local and distant clusters (e.g., Melnick \& Sargent 1977; Dressler 1980; Whitmore et al. 1993; Abraham et al. 1996; Dressler et al. 1999; Gerken et al. 2004).

In a second-order analysis, we applied the 2DKS-test to compare the distributions of projected positions. This is particularly meaningful for the MACS1206 dataset, which is large enough to search for differences in the internal structure. This test is more sensitive than the above $1 \mathrm{DKS}$-test and, in addition to confirming the above dichotomy, it is able to detect differences within the ELG class, specifically between mELG and vsELG, and between sELG and vsELG (marginally, at the $<95 \%$ c.l.). The combined $\mathrm{mELG}+\mathrm{sELG}$ class differs from vsELG at the $96 \%$ c.l. The observed difference is related to the cluster substructure and will be further analyzed and discussed in following sections.

The application of the KW-test to the rest-frame velocities $V_{\text {rf }}$ [i.e., $\left.(V-\langle V\rangle) /(1+z)\right]$ gives no significant result, i.e., the velocity distributions of different spectral classes can originate 


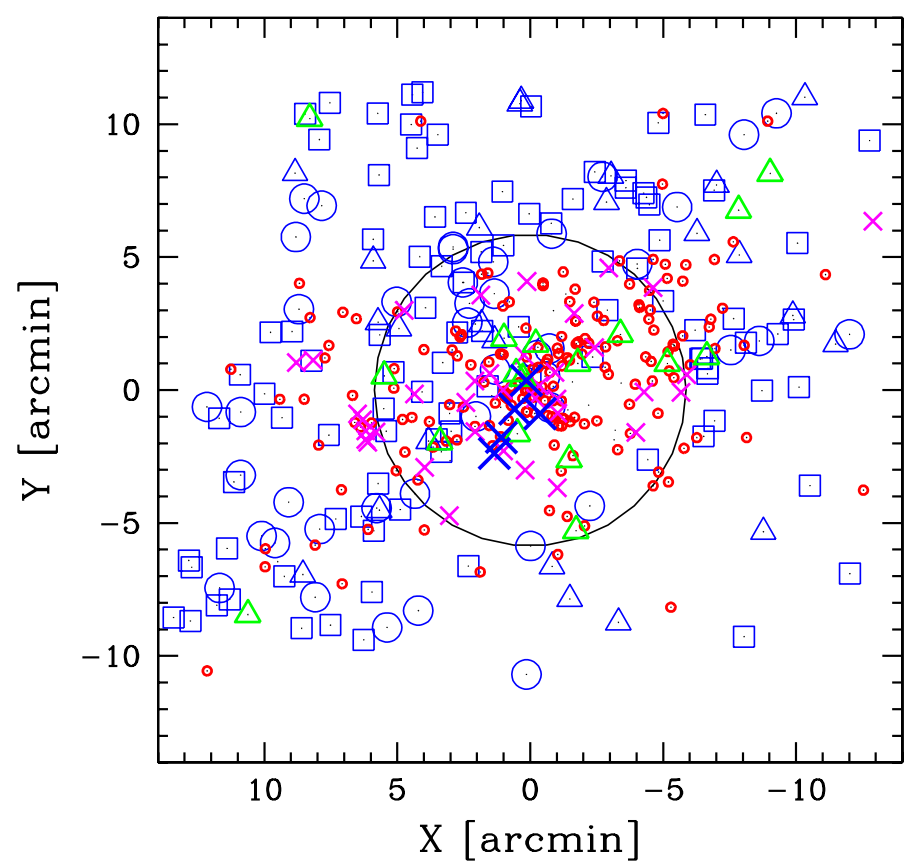

Fig. 4. Spatial distribution of the 445 cluster members highlighting the spatial segregation between early- and late-type galaxies across our spectral classes. Each classified galaxy is marked by a symbol: PAS (small red circles); HDSr (magenta crosses); HDSb (large blue crosses); wELG (green triangles); mELG (blue triangles); sELG (blue squares); vsELG (blue circles). The circle centered on the BCG encloses the $R_{200}$ region.

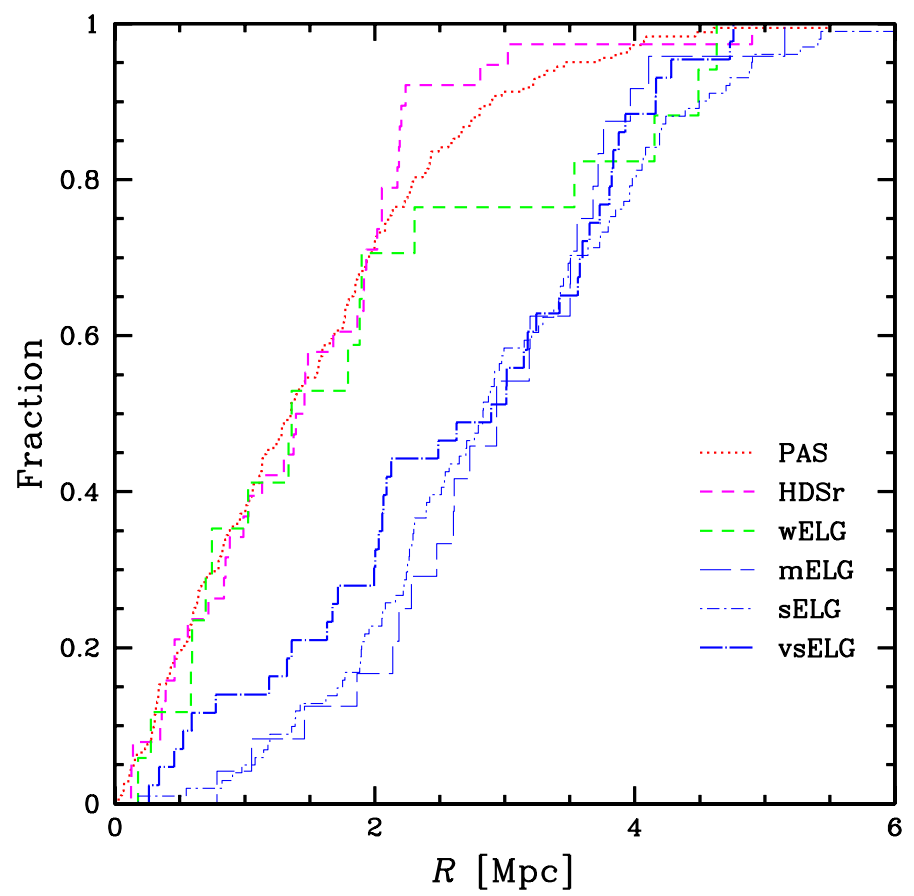

Fig. 5. Cumulative distributions of clustercentric distance $R$ of galaxies per spectral class, showing the spatial segregation from early- through late-type galaxies.

from the same parent distribution (see Fig. 6, upper panel). We also applied the KW-test to the absolute value of $V_{\text {rf }}$ in such a way to be more specifically sensitive to differences in $\sigma_{V}$ values: no significant result was obtained (see Fig. 6, lower panel). When comparing two by two the $\langle V\rangle$ estimates of different
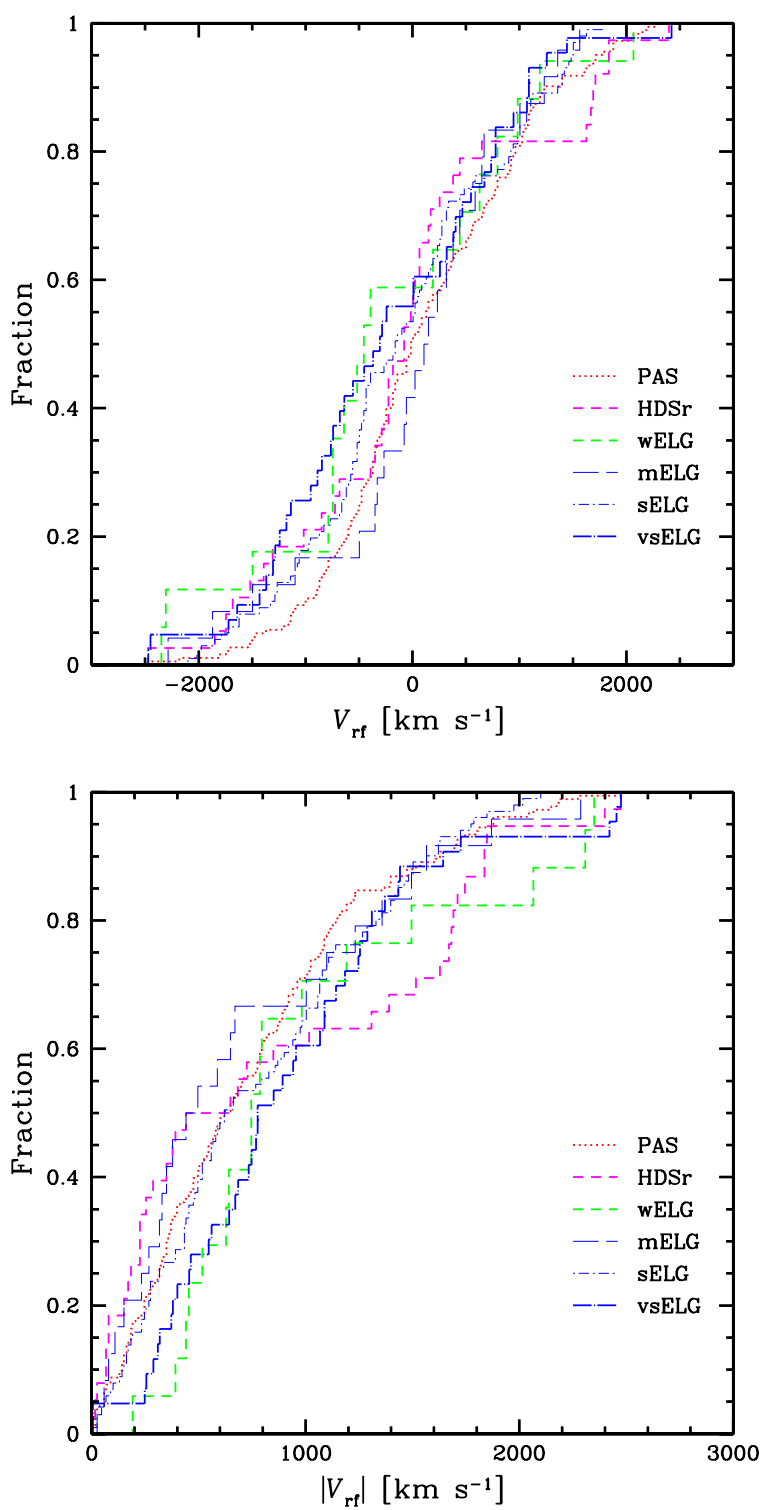

Fig. 6. Cumulative distributions of rest-frame LOS velocity $V_{\mathrm{rf}}$ and absolute $V_{\mathrm{rf}}$ of galaxies per spectral class (upper and lower panels, respectively).

spectral classes (see Table 3), we found no significant difference. The same is true for $\sigma_{V}$. Our results corroborate previous studies claiming for a velocity distribution which does not depend dramatically on galaxy color or spectral type (Rines et al. 2005, 2013; Mahajan et al. 2011), including the results of B13 on MACS1206 itself. On the other hand, several previous studies have found significant differences in the velocity distributions of different galaxy populations, the velocity dispersion of the population of blue SF galaxies being higher than that of the population of red passive galaxies (e.g., Tammann 1972; Moss \& Dickens 1977; Sodré et al. 1989; Biviano et al. 1992, 1997; Zabludoff \& Franx 1993; Colless \& Dunn 1996; Adami et al. 1998; Dressler et al. 1999). The cause for this controversy may lie in i) the selection criteria of member galaxies of past studies, which were often not based on extensive spectroscopic information; ii) the dynamical status of the analyzed clusters; or iii) an evolutionary trend when considering clusters at different redshifts. We defer a more complete analysis of this question when our analysis has been extended to other CLASH-VLT clusters. 
Table 3. Kinematical properties of the whole cluster and galaxy populations.

\begin{tabular}{lrlr}
\hline \hline Sample & $N_{\mathrm{g}}$ & $\begin{array}{c}\langle V\rangle \\
\mathrm{km} \mathrm{s}^{-1}\end{array}$ & $\begin{array}{c}\sigma_{V} \\
\mathrm{~km} \mathrm{~s}^{-1}\end{array}$ \\
\hline TOT & 445 & $131843 \pm 49$ & $1035_{-45}^{+27}$ \\
R200 & 249 & $131776 \pm 74$ & $1169_{-52}^{+54}$ \\
PAS & 183 & $131980 \pm 69$ & $940_{-85}^{+76}$ \\
HDSr & 38 & $131630 \pm 196$ & $1188_{-379}^{+249}$ \\
HDSb & 5 & $132836 \pm 767$ & $1307_{-141}^{+919}$ \\
wELG & 17 & $131502 \pm 307$ & $1215_{-363}^{+415}$ \\
ELG & 168 & $131690 \pm 78$ & $1014_{-83}^{+83}$ \\
mELG & 24 & $132121 \pm 192$ & $917_{-299}^{+366}$ \\
sELG & 101 & $131698 \pm 99$ & $996_{-100}^{+90}$ \\
vsELG & 43 & $131485 \pm 170$ & $1102_{-161}^{+235}$ \\
\hline
\end{tabular}

Notes. Column 1: sample ID. ${ }^{(a)}$ The ELG class is formed of mELG, sELG, and vsELG); Col. 2: the number of assigned galaxies, $N_{\mathrm{g}}$; Cols. 3 and 4: mean LOS velocity and velocity dispersion of galaxies, $\langle V\rangle$ and $\sigma_{V}$, with respective errors.

The specific case of MACS1206 is further analyzed at the end of this section.

The spatial separation between PAS and ELG galaxies is also clear in Fig. 7 (top panel), where we show the distribution of galaxies of different spectral classes in the plot of rest-frame velocity vs. projected clustercentric distance, the so-called projected phase-space diagram. In the same figure we also plot the integral and differential $\langle V\rangle$ and $\sigma_{V}$ profiles (middle and bottom panels). The integral $\langle V\rangle$ profile shows that the measure of the mean velocity is independent of the limiting radius of the sampled region in agreement with a scenario of a unimodal, relaxed cluster. As for the differential $\langle V\rangle$ profiles, the most noticeable feature is the small value of $\langle V\rangle$ of the ELG and TOT populations at $R \lesssim 2 h_{70}^{-1} \mathrm{Mpc}$. This feature is likely related to the presence of a low-velocity substructure detected at $\lesssim R_{200}$ in the NE quadrant (see Sect. 4.3).

The integral and differential $\sigma_{V}$ profiles show that the velocity dispersion decreases with $R$. This trend is the result of the cluster mass density profile and the anisotropy velocity profile as discussed in B13. The interesting feature is that the face values of $\sigma_{V}$ as computed for the ELG population are higher than those of the PAS population at comparable radii. This is also shown in Fig. 3 of B13, where the two populations are color defined. However, the errors in individual bins are large and the difference is scarcely significant. To further investigate this point we used two approaches. We fitted the velocity dispersion profiles of ELG and PAS galaxies as given in Fig. 7 (bottom panel) obtaining $\lg \left(\sigma_{V, \text { ELG }}\right)=3.15( \pm 0.124)-0.399( \pm 0.135) \times \lg (R)$ and $\lg \left(\sigma_{V, \text { PAS }}\right)=2.98( \pm 0.121)-0.161( \pm 0.047) \times \lg (R)$, where $\sigma_{V}$ and $R$ are in units of $\mathrm{km} \mathrm{s}^{-1}$ and $h_{70}^{-1} \mathrm{Mpc}$. The slopes are different at the $92 \%$ c.l. according to the Welch test (e.g., Guest 1961). In the second approach we considered the region between 1 and $3 h_{70}^{-1} \mathrm{Mpc}$, where both populations are well represented, obtaining $\sigma_{V, \mathrm{ELG}, 1-3}=1085_{-68}^{+67}$ for 99 ELG galaxies and $\sigma_{V, \mathrm{PAS}, 1-3}=$ $857_{-58}^{+56}$ for 82 PAS galaxies, i.e., $\sigma_{V, \text { ELG,1-3 }}>\sigma_{V, \text { PAS, } 1-3}$ at the $97 \%$ c.l. according to the F-test (see, e.g., Press et al. 1992). We conclude that, in the case of MACS1206, the PAS and ELG populations do differ in their velocity dispersion but this difference is hidden by the combination of the trend of the $\sigma_{V}$ profile with the strong PAS vs. ELG spatial segregation when global values are analyzed (see the first part of this section).

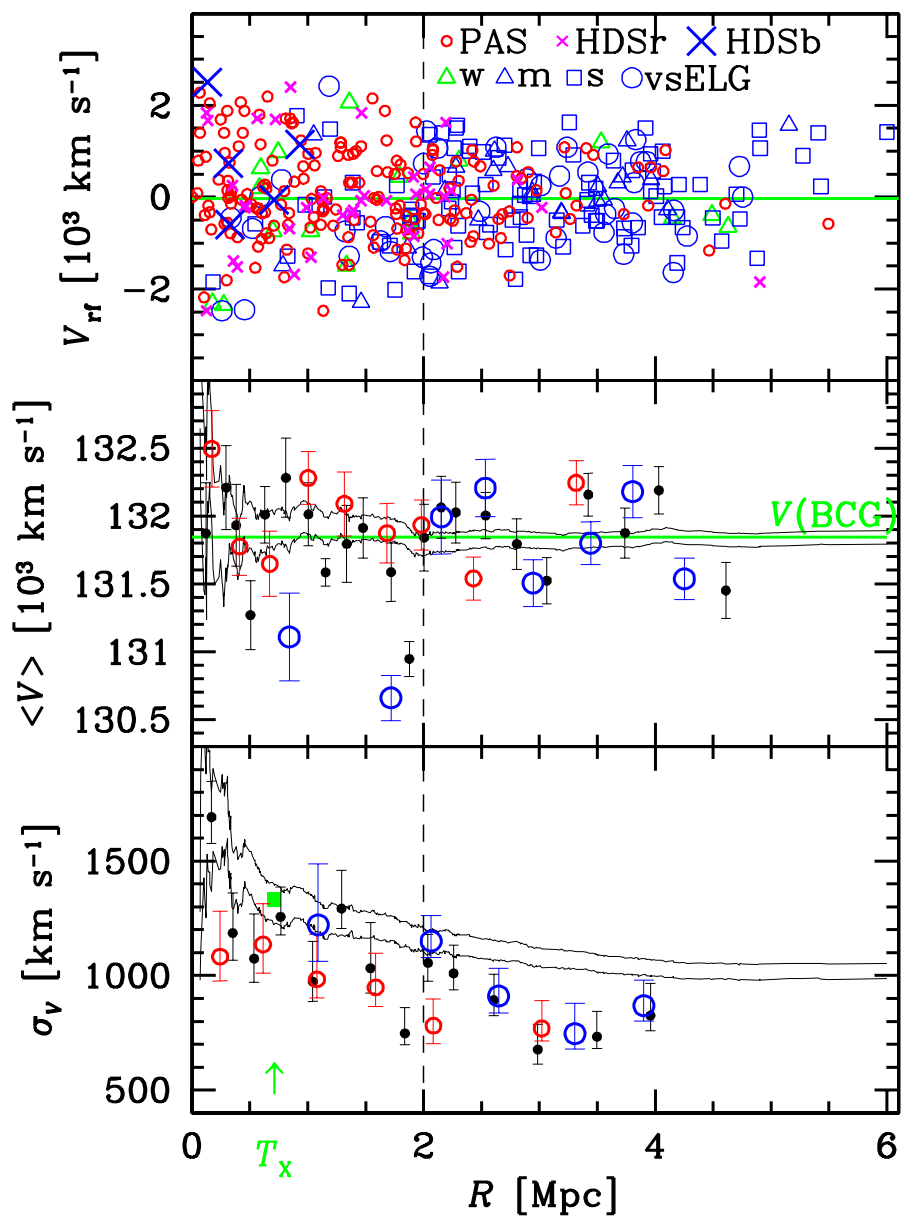

Fig. 7. Top panel: rest-frame LOS velocity vs. projected clustercentric distance for galaxies of different spectral classes. The cluster center coincides with the position of the BCG and the vertical dashed line indicates the value of $R_{200}$. The horizontal green line indicates the BCG velocity. Middle panel: integral profile of the mean LOS velocity shown through its error bands at the $68 \%$ c.l. The mean velocity at a given projected radius from the cluster center is estimated by considering all galaxies within that radius. Small black dots, small red circles, and blue circles show the differential profiles for TOT, PAS, and ELG galaxies (each point is the value computed using 20 galaxies). Bottom panel: as in the middle panel, but for the LOS velocity dispersion (here each point is the value computed using 30 galaxies). The green square indicates the estimate of the X-ray temperature $T_{\mathrm{X}}$ reported by Postman et al. (2012) using Chandra data within the $R=0.714 h_{70}^{-1} \mathrm{Mpc}$ radius, indicated by the position of the green arrow, and here converted to $\sigma_{V}$ assuming the density-energy equipartition between ICM and galaxies, i.e., $\beta_{\text {spec }}=1$ (see Sect. 5.1).

\section{Cluster substructure}

We applied a set of tests in the velocity space (1D-tests), in the 2D space of positions projected on the sky, and in the combined $2 \mathrm{D}+1 \mathrm{D}$ space (3D-tests). Since the sensitivity of individual diagnostics depends on the relative position and velocity of substructure, no single substructure test is the most sensitive in all situations (Pinkney et al. 1996).

\subsection{Analysis of the velocity distribution}

The velocity distribution was analyzed to search for possible deviations from Gaussianity that might provide important signatures of complex internal dynamics. We used the two robust shape estimators by Bird \& Beers (1993), the asymmetry index 
Table 4. Results of the substructure analysis.

\begin{tabular}{|c|c|c|c|c|c|c|c|c|c|}
\hline \multirow[b]{2}{*}{ Sample } & \multirow[b]{2}{*}{$N_{\mathrm{g}}$} & \multicolumn{4}{|c|}{ 1D } & \multirow{2}{*}{$\begin{array}{c}2 \mathrm{D} \\
\text { DED. } \\
N_{\mathrm{p}}\end{array}$} & \multicolumn{3}{|c|}{$2 \mathrm{D}+1 \mathrm{D}$} \\
\hline & & $\begin{array}{l}\text { TI } \\
\%\end{array}$ & $\begin{array}{l}\mathrm{AI} \\
\%\end{array}$ & $\begin{array}{c}V_{\mathrm{BCG}} \text { pec. } \\
\% \\
\end{array}$ & $\begin{array}{c}\text { DED. } \\
N_{\mathrm{p}}\end{array}$ & & $\begin{array}{c}V \text { grad. } \\
\%\end{array}$ & $\begin{array}{l}\mathrm{DS}\langle V\rangle \\
\%\end{array}$ & $\underset{\%}{\mathrm{DS} \sigma_{V \text {,corr }}}\left(\mathrm{DS} \sigma_{V}\right)$ \\
\hline WHOLE & 445 & ns & ns & ns & 1 & $1+3$ & ns & ns & ns $(>99.9)$ \\
\hline R200 & 249 & ns & ns & ns & 1 & $1+1$ & ns & $\mathrm{ns}$ & ns (ns) \\
\hline PAS & 183 & $90-95$ & ns & ns & 1 & $1+1$ & ns & ns & $94(92)$ \\
\hline $\mathrm{HDSr}$ & 38 & 90-95 & 95-99 & - & 1 & 2 & ns & ns & ns (ns) \\
\hline wELG & 17 & ns & ns & - & - & 1 & $\mathrm{~ns}$ & - & - \\
\hline ELG & 168 & $90-95$ & ns & - & 1 & 7 & ns & $>99.9$ & ns (99.7) \\
\hline vsELG & 43 & ns & ns & - & 1 & 2 & $\mathrm{~ns}$ & 99.1 & ns (94) \\
\hline
\end{tabular}

Notes. Column 1: sample ID; Col. 2: number of galaxies, $N_{\mathrm{g}}$; Cols. 3 and 4: significance of the deviations from Gaussian according to the tail and asymmetry indices; Col. 5: significance of the peculiarity of the BCG velocity; Col. 6: number of peaks detected through the 1D-DEDICA method, only peaks detected with a c.l. larger than $99 \%$ are considered; Col. 7: number of peaks detected through the 2D-DEDICA method, where n1+n2 indicates the presence of $\mathrm{n} 1$ major peaks and $\mathrm{n} 2$ minor with very low-density peaks; Col. 8: significance of the existence of a velocity gradient; Cols. 9 and 10: significance of the existence of substructure according to the DS $\langle V\rangle$-test and the DS $\sigma_{V}$-test, the latter for both the profile-corrected and standard versions. Only significance values larger than $90 \%$ are reported, while non-significant values are indicated with ns.

(AI) and the tail index (TI). Following the Indicator test by Gebhardt \& Beers (1991), we also checked the peculiarity of the BCG velocity $\left(V_{\mathrm{BCG}}=131849 \mathrm{~km} \mathrm{~s}^{-1}\right)$ in all the samples containing the BCG (namely the TOT, R200, and PAS samples).

To detect and analyze possible departures from a single-peak distribution, we used the 1D-DEDICA method, already applied in Sect. 2 to determine MACS1206 membership. DEDICA was introduced by Pisani (1993), and extended to the multivariate case in Pisani (1996). The algorithm is described in the original studies and references therein. Here we summarize useful points (see also Appendix A in Girardi et al. 1996). DEDICA is a method of cluster analysis based on the estimate of the probability density of a data sample, where the density is estimated by using an iterative and adaptive kernel estimator, in this case a Gaussian kernel with an optimal choice of the width. It is based on the assumption that a cluster corresponds to a local maximum in the density of points. This method allows us to estimate i) the statistical significance of each subcluster it detects and ii) the probability that each galaxy in the data sample is a member of each detected subcluster. In particular, it gives a measure of the overlapping between two systems (see Girardi et al. 1996; Pisani et al. 1996). We note that DEDICA is a non-parametric method in the sense that it does not require any assumption about the number of clusters or any other of their features. Table 4 summarizes the results of the 1D-DEDICA method applied to the TOT and other samples of MACS1206 and Fig. 8 shows the relevant plots.

The velocity distributions of the TOT and R200 samples show no departures from a Gaussian, nor signs of a peculiar BCG velocity, and only one significant peak is detected by the 1D-DEDICA reconstruction. Within $0.5 R_{200}$, two peaks, formed of 91 and 37 galaxies, are detected by the 1D-DEDICA reconstruction (see Fig. 8). However, the two groups are strongly overlapped with $26+26$ galaxies having a non-negligible probability to belong to both groups and not different in their 2D distributions.

The velocity distributions of galaxy populations per spectral class show marginal or no departures from a Gaussian with the exception of the HDSr population. In the case of the PAS class no sign of a peculiar BCG velocity is found. Moreover, only one significant peak is detected by the 1D-DEDICA reconstruction with the exception of wELG, a very poor class where a nonsignificant peak can be detected. The velocity distribution of the PAS galaxies outside $R_{200}$ and that of the PAS galaxies within

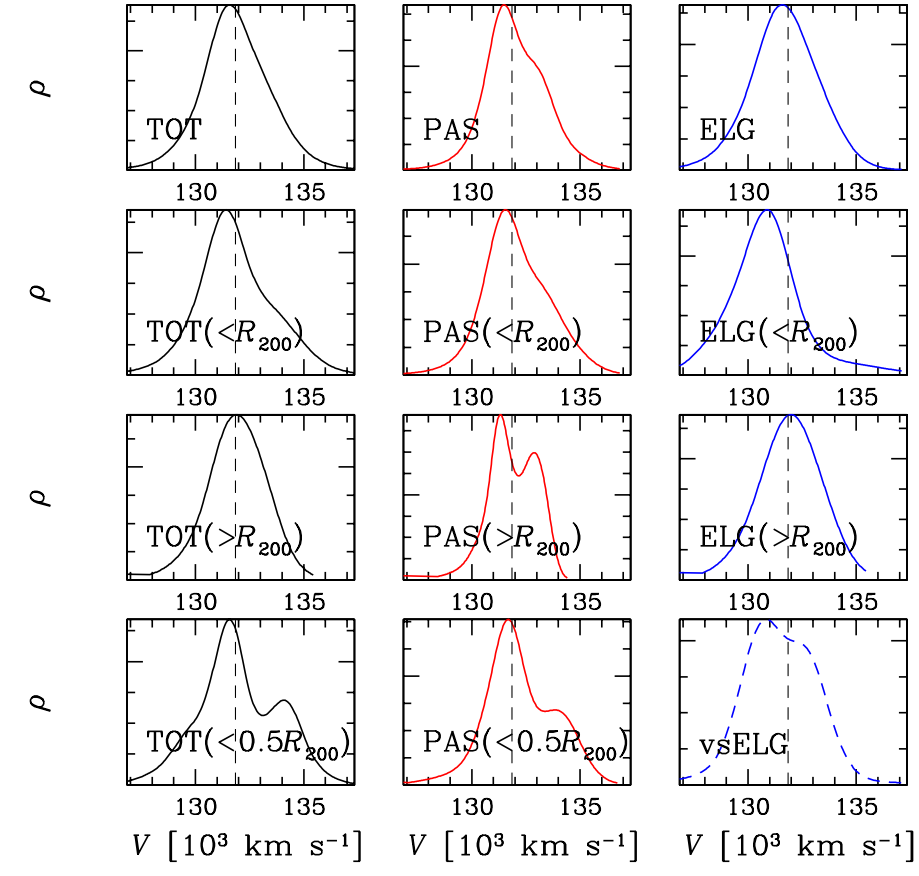

Fig. 8. Galaxy density reconstruction in the LOS velocity space through the 1D-DEDICA method. Results for all galaxies in the TOT, PAS, and ELG samples are shown from left to right, while results for the whole and partial cluster regions are shown from top to bottom. The result in the vsELG sample is shown in the bottom-right panel. In each panel, the dashed vertical line indicates the BCG velocity. Units on the $y$-axis are rescaled to the maximum value of each density distribution.

$0.5 R_{200}$ show the presence of two peaks, but, as in the case discussed above, the two groups are strongly overlapped and show no difference in their spatial distribution.

We note the peculiar velocity distribution of ELGs within $R_{200}$, which is significantly peaked at lower values with respect to the mean velocity of the whole cluster, $\left\langle V_{\mathrm{ELG},<\mathrm{R} 200}\right\rangle=$ $(130709 \pm 171) \mathrm{km} \mathrm{s}^{-1}$. The velocity distribution of vsELGs is peaked around the same value and, in addition, the visual inspection of the 1D-DEDICA reconstruction (Fig. 8, bottom-right panel) suggests a secondary bump at high velocities. These features are related to the presence of a low-velocity NE galaxy structure and of a high-velocity SEext galaxy structure as we 


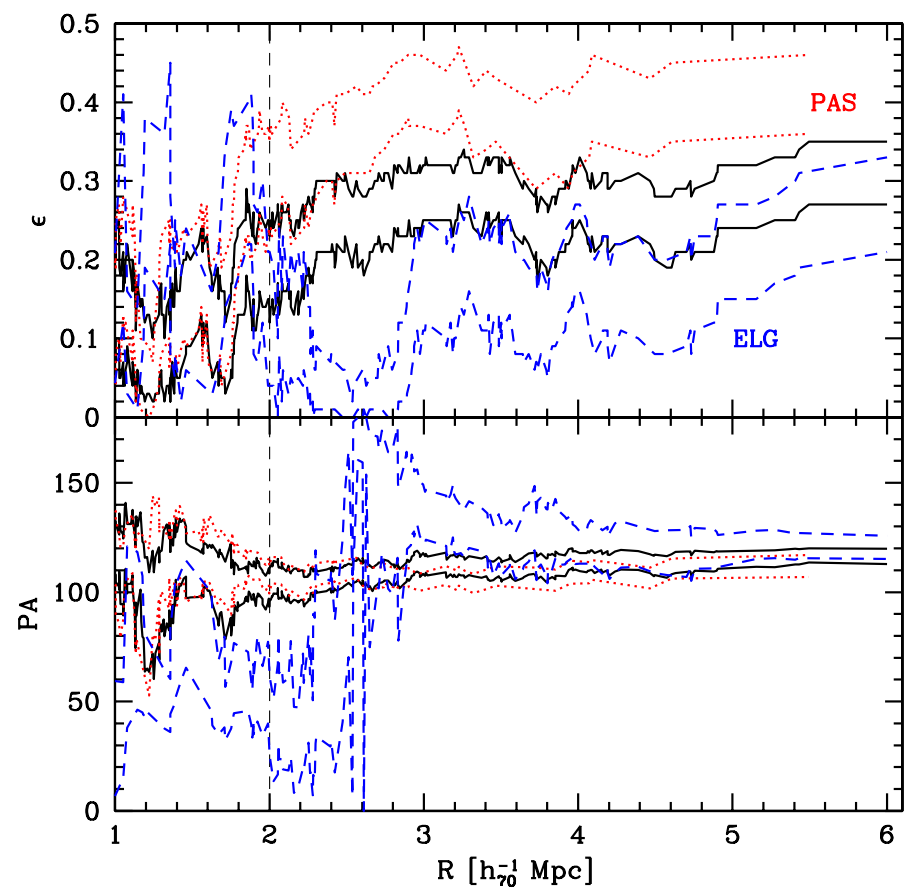

Fig. 9. Integral profiles of ellipticity (upper panel) and position angle (lower panel) for the whole galaxy population (solid black line) and per spectral class according to the labels in the upper panel. The values of $\epsilon$ and PA at a given radius $R$ are estimated by considering all galaxies within $R$. Results within $1 h_{70}^{-1} \mathrm{Mpc}$ are too noisy to be meaningful. The vertical dashed line indicates the value of $R_{200}$.

discuss in Sect. 4.3. We do not plot the reconstructions for HDSr and wELG, whose distributions are poorly sampled.

\subsection{Analysis of the $2 D$ galaxy distribution}

The main features in Fig. 4 are the WNW-ESE elongation and the lack of ELGs in the SW quadrant with respect to the NE quadrant (14 vs. 54 galaxies). In the computation of ellipticity $(\epsilon)$ and position angle of the major axis (PA), we followed the moments of inertia method (Carter \& Metcalfe 1980; see also Plionis \& Basilakos 2002 with weight $w=1)$ ). Table 5 lists the values of $\epsilon$ and PA (measured counterclockwise from north) for the TOT and R200 samples, and the main spectral classes. Figure 9 shows the integral estimates of $\epsilon$ and PA at increasing radii. As far as concerning PAS galaxies, the value of $\epsilon$ increases out to $R \sim R_{200}$, which is the radius containing most PASs, and then is stable. The value of PA is already stable at $R \lesssim R_{200}$. As for ELGs, Fig. 9 allows us to appreciate a strong variation of $\epsilon$ and PA at $R \sim 2-3 R_{200}$, while values are roughly stable at $R \gtrsim 3 h_{70}^{-1} \mathrm{Mpc}$. This variation is likely due to the NE vs. SW quadrant asymmetry and, specifically, to the presence of an important NE structure as discussed at the end of the section. When comparing values at large radii, $R \gtrsim 3 h_{70}^{-1} \mathrm{Mpc}$, the PAS and ELG spatial distributions have similar position angles, PA $=110-120^{\circ}$, while the ELG distribution is somewhat rounder than the PAS distribution, $\epsilon_{\mathrm{PAS}} \sim 1.5-2 \epsilon_{\mathrm{ELG}}$ at the $2-3 \sigma$ c.l. depending on the precise radius. Table 5 also lists the values of $\epsilon$ and PA for the HDSr population, but the respective profiles are not shown in Fig. 9 for the sake of clarity. The value of $\mathrm{PA}_{\mathrm{HDSr}}$ agrees with that of PAPAS within $1 \sigma$ c.l. The value of $\epsilon_{\mathrm{HDSr}}$ is larger than $\epsilon_{\mathrm{PAS}}$ only at a $\$ 2 \sigma$ c.l., the large value of $\epsilon_{\mathrm{HDSr}}$ being due to the few galaxies of the dense ESE peak (see in the following).
Table 5. Ellipticity and position angle of the galaxy distribution.

\begin{tabular}{lcc}
\hline \hline Sample & $\epsilon$ & $\begin{array}{c}\text { PA } \\
\text { deg }\end{array}$ \\
\hline TOT & $0.31_{-0.04}^{+0.04}$ & $117_{-4}^{+3}$ \\
R200 & $0.20_{-0.06}^{+0.05}$ & $102_{-8}^{+7}$ \\
PAS & $0.41_{-0.05}^{+0.05}$ & $111_{-4}^{+6}$ \\
HDSr & $0.55_{-0.06}^{+0.07}$ & $105_{-4}^{+8}$ \\
ELG & $0.26_{-0.05}^{+0.07}$ & $121_{-6}^{+5}$ \\
\hline
\end{tabular}

Table 6. Results of the 2D-DEDICA analysis.

\begin{tabular}{lrcrrr}
\hline \hline Subclump & $N_{\mathrm{S}}$ & $\begin{array}{c}\alpha(\mathrm{J} 2000), \delta(\mathrm{J} 2000) \\
\mathrm{h}: \mathrm{m}: \mathrm{s}^{\circ} \mathrm{:}^{\prime}:^{\prime \prime}\end{array}$ & $\rho_{\mathrm{S}}$ & $\chi_{\mathrm{S}}^{2}$ \\
\hline TOT-main & 219 & $120611.5-084757$ & 1.00 & 103 \\
TOT-WNW & 102 & $120549.4-084644$ & 0.20 & 29 \\
TOT-SEint & 38 & $120624.9-084937$ & 0.20 & 16 \\
(TOT-ESE) & 41 & $120635.0-084926$ & 0.15 & 15 \\
\hline R200-main & 203 & $120611.5-084757$ & 1.00 & 80 \\
R200-SEint & 30 & $120624.8-084940$ & 0.20 & 17 \\
\hline PAS-main & 126 & $120612.2-084805$ & 1.00 & 41 \\
PAS-WNW & 44 & $120551.0-084630$ & 0.25 & 12 \\
\hline (HDSr-ESE) & 6 & $120637.0-084937$ & 1.00 & 6 \\
HDSr-main & 15 & $120611.0-084757$ & 0.67 & 4 \\
\hline wELG & 15 & $120612.0-084720$ & 1.00 & 7 \\
\hline ELG-NE & 39 & $120621.8-084400$ & 1.00 & 14 \\
ELG-NW & 30 & $120557.2-084038$ & 0.85 & 11 \\
ELG-WNW & 24 & $120542.0-084634$ & 0.68 & 9 \\
ELG-SE & 13 & $120636.0-085234$ & 0.58 & 5 \\
ELG-SEext & 20 & $120649.7-085419$ & 0.43 & 10 \\
ELG-NEext & 17 & $120631.0-083810$ & 0.31 & 8 \\
ELG-ESEext & 10 & $120654.2-084828$ & 0.31 & 5 \\
\hline vsELG-NE & 24 & $120620.5-084420$ & 1.00 & 9 \\
vSELG-SEext & 15 & $120649.3-085313$ & 0.55 & 4 \\
\hline
\end{tabular}

Notes. Column. 1: subsample/peak ID, labels in parentheses correspond to galaxy peaks that do not completely fulfil the criteria we fixed for galaxy number or density, but are listed here becasue they are discussed in the text; Col. 2: number of assigned member galaxies, $N_{\mathrm{S}}$; Col. 3: RA and Dec of the density peak; Col. 4: relative density with respect to the highest peak, $\rho_{\mathrm{S}}$; Col. 5: $\chi^{2}$ value of the peak. For each population, the number of peaks is summarized in Table 4 (Col. 7).

We also analyzed the galaxy spatial distribution through the 2D-DEDICA analysis (Pisani 1996). In Table 6, we present the full information for the relevant peaks, i.e., those with a c.l. $\geq 99 \%$, with a relative density with respect to the main peak $\rho_{\mathrm{S}} \gtrsim 0.20$, and with at least 10 assigned galaxies. The relevant maps are shown in Figs. 10 and 11. When analyzing the whole cluster, the 2D-DEDICA density reconstruction confirms the cluster elongation and shows a main structure peaked on the BCG with a few secondary peaks (at ESE, SE, and WNW). The SE peak in the internal cluster region (hereafter SEint), with 38 assigned galaxies, is the only secondary peak detected in the R200 sample.

When considering PAS galaxies, the main feature is the clear elongation along the WNW-ESE direction in the plane of the sky, with the presence of an external secondary peak at WNW. Noticeably, HDSr galaxies trace a very dense peak $\sim 2 h_{70}^{-1} \mathrm{Mpc}$ at ESE, denser than that around the cluster center. This ESE peak is formed of only six galaxies, but its existence is supported by our independent analysis in Sect. 4.4 based on 


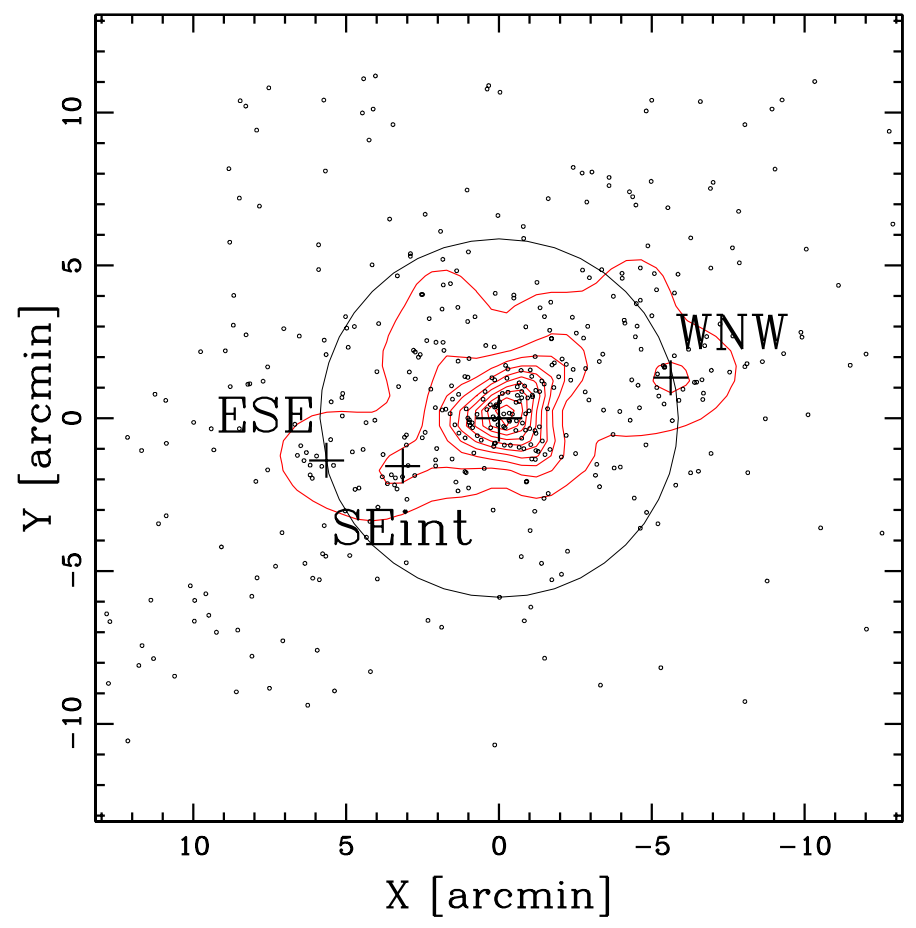

Fig. 10. Spatial distribution and isodensity contours of the 445 cluster members (TOT sample). Large cross and circle highlight the BCG position and the $R_{200}$ region. Small crosses indicate the secondary density peaks (see Table 6).

galaxies of different colors. We detected no peculiarity in the velocity field in the ESE region. The 2D-DEDICA analysis of the wELG population shows only one peak roughly centered on the cluster center. To the contrary, the 2D distribution of ELGs is clearly not radially symmetric, with a lack of galaxies in the SW region and shows several clumps (see Table 6). The ELG distribution shown in Fig. 11 allows us to better interpret the variation of $\epsilon$ and PA in Fig. 9. The NE structure centered at $R \lesssim R_{200}$ is the cause of the value of $\mathrm{PA} \sim 50^{\circ}$ at $R \sim R_{200}$. When more external ELG structures, somewhat related to the NE-SW direction, are included the PA value shows a fast, strong change and the $\epsilon$ value also increases. According to our analysis in Sect. 3.2, the spatial distribution of vsELGs is different from that of mELGs and sELGs. The reason is that vsELGs are concentrated in the NE and SEext structures, as shown by the contour maps for vsELGs and mELGs+sELG galaxies (see Fig. 12).

The study of Pisani (1996) shows that the 2D-DEDICA method, based on the centrally symmetric two dimensional Gaussian kernel, can reconstruct the density of an elongated $2 \mathrm{D}$ system even in the case with an axial ratio $a / b=0.1$, although slightly rounder isoplethes are obtained (see his Fig. 2). Pisani (1996) also shows that a simulated cluster with $a / b=0.1$ and strongly contaminated by a population of randomly distributed points ( 100 random points and 100 cluster points) is split into a series of clusters strongly overlapping each other along the major axis (see his Fig. 4 and Table 1). This second case is very extreme with respect to MACS1206 both for the presence of an important background and the high ellipticity (cf. $a / b=0.1$ and $a / b=0.7)$. However, to leave no room for doubt in the case of MACS1206, we run an additional test. We performed the circularization of the coordinates in the TOT sample, leaving the cluster center fixed and using the ellipticity listed in Table 5, and repeated the full 2D-DEDICA analysis. We confirmed the presence and the relative density of the main WNW and ESE peaks, but not the SEint peak. However, the SEint peak and its relative density are confirmed in the re-analysis of the R200 sample after the circularization procedure. The WNW and ESE peaks are also independently confirmed in the re-analysis of the PAS and HDSr samples.

\subsection{Combining position and velocity information}

The existence of correlations between positions and velocities of cluster galaxies is always a strong footprint of real substructures. To study the 3D cluster structure we applied two tests. The presence of a velocity gradient was searched for by performing a multiple linear regression fit to the observed velocities with respect to the galaxy positions in the plane of the sky (e.g., den Hartog \& Katgert 1996; Girardi et al. 1996). The significance is based on 1000 Monte Carlo simulated clusters obtained by shuffling galaxy velocities with respect to their positions. We found no significant evidence for a velocity gradient.

The $\Delta$-statistics devised by Dressler \& Schectman (1988, hereafter DS-test) is a powerful test for $3 \mathrm{D}$ substructure, which is valid in samples down to 30 member galaxies, at least in the case of major mergers (Pinkney et al. 1996). For each galaxy, the deviation $\delta_{i}$ is defined as $\delta_{i}^{2}=\left[\left(N_{\mathrm{nn}}+1\right) / \sigma_{V}^{2}\right]\left[\left(\langle V\rangle_{\mathrm{loc}}-\langle V\rangle\right)^{2}+\right.$ $\left.\left(\sigma_{V, \text { loc }}-\sigma_{V}\right)^{2}\right]$, where the subscript loc denotes the local quantities computed over the $N_{\mathrm{nn}}=10$ neighbors of the galaxy, $\langle V\rangle$ and $\sigma_{V}$ are the global quantities, and $\Delta$ is the sum of the $\left|\delta_{i}\right|$ of the individual $N$ galaxies of the sample. The significance of $\Delta$, i.e., of the existence of substructure, is checked by running 1000 Monte Carlo simulations, randomly shuffling the galaxy velocities. Here, we used two kinematical estimators alternative to the $\delta_{i}$ parameter. We considered separately the contribution of the deviation of the local mean velocity from global mean velocity $\delta_{i, V}=\left[\left(N_{\mathrm{nn}}+1\right)^{1 / 2} / \sigma_{V}\right] \times\left(\langle V\rangle_{\mathrm{loc}}-\langle V\rangle\right)$ and the contribution of the deviation of the local velocity dispersion from the global velocity dispersion $\delta_{i, \mathrm{~s}}=\left[\left(N_{\mathrm{nn}}+1\right)^{1 / 2} / \sigma_{V}\right] \times\left(\sigma_{V \text {,loc }}-\sigma_{V}\right)$ (Barrena et al. 2011; see also Girardi et al. 1997). The results are listed in Table 4.

The DS $\langle V\rangle$-test returns a strong positive detection of substructure in the case of the ELG population (>99.9\% c.1.), with a low-velocity group within $R_{200}$ at $\mathrm{NE}$ and a high-velocity group in the external SE region (see Fig. 13 for the so-called bubble plot). To be more quantitative, we resorted to the technique developed by Biviano et al. (2002; see also Girardi et al. 2006). We compared the distribution of the $\delta_{i, V}$ values of the real ELG galaxies to the distribution of the $\delta_{i, V}$ values of the galaxies of all the 1000 Monte Carlo simulated ELG samples (Fig. 14). In agreement with the result of the DS $\langle V\rangle$-test, the two distributions differ at the $>99.99 \%$ c.l. according to the 1DKS-test. The distribution of the values of real galaxies shows a tail at large positive and negative $\delta_{i, V}$ values, populated by the galaxies belonging to the $3 \mathrm{D}$ substructure. In particular, the low-velocity tail is more populated than the high-velocity tail in agreement with the relative importance of the low-velocity NE substructure in Fig. 13. To quantify the number of galaxies involved in the 3D substructure, we assumed a threshold of $\left|\delta_{i, V}\right|=3$ and counted 46 real galaxies with $\left|\delta_{i, V}\right|>3$, while five galaxies are predicted by simulations; in other words, $\sim 41$ ELGs are expected to belong to the $3 \mathrm{D}$ substructure. The galaxies with $\left|\delta_{i, V}\right|>3$ are spatially separated in the NE and SE clumps allowing us to estimate the rest-frame velocity of these clumps, $\Delta V_{\mathrm{rf}, \mathrm{NE}} \sim-550 \mathrm{~km} \mathrm{~s}^{-1}$ and $\Delta V_{\text {rf,SEext }} \sim+550 \mathrm{~km} \mathrm{~s}^{-1}$.

We note that the tails of the ELG $\delta_{i, V}$ distribution are populated by several vsELGs; 20 vsELGs, i.e., about half of the 

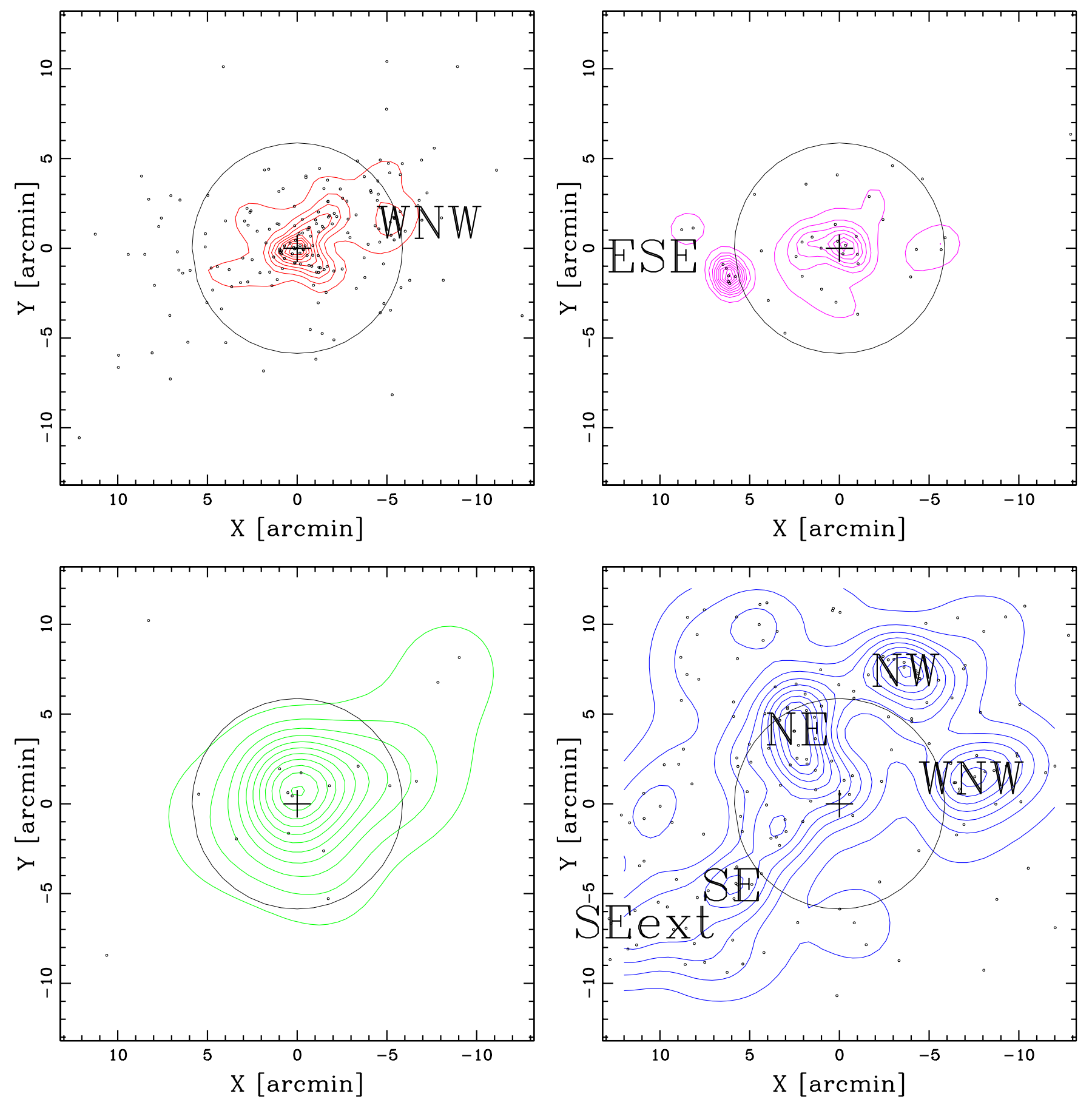

Fig. 11. Spatial distributions and isodensity contour maps for the PAS (upper left panel), HDSr (upper right panel), wELG (lower left panel), and ELG (lower right panel) populations. Labels refer to the peaks listed in Table 6 . The $R_{200}$ region is highlighted by the circle centered on the BCG.

whole vsELG population, have $\left|\delta_{i, V}\right|>2.5$. This prompted us to apply the DS $\langle V\rangle$-test to the ELG subclasses. The test applied to the vsELG class returns, in spite of the small number of vsELG galaxies, a $99.1 \%$ c.l. signal of substructure (see Fig. 15), while no signal is found for mELG and sELG. From these results and those of the previous section we can conclude that vsELGs are strongly concentrated in the NE and SEext substructures and are related to the $3 \mathrm{D}$ substructure.

In the context of the above results, the features in the velocity distributions of ELGs and vsELGs plotted in Fig. 8 can be explained. The velocity distribution of ELGs within $R_{200}$ is peaked at low velocity owing to the low-velocity NE substructure, very important within $R_{200}$ (see Fig. 13). In the case of vsELGs (whole sample), the high-velocity SEext substructure is also important resulting in a velocity distribution suggestive of two peaks.

As for the DS $\sigma_{V}$-test, the MACS1206 dataset is so large in galaxy content and in sampled area that the radial $\sigma_{V}$ profile (Fig. 7, bottom panel; see also Fig. 3 in B13) should be taken into account. The application of the standard DS $\sigma_{V}$-test in the TOT sample detects a spurious strong signal of substructure ( $>99.9 \%$ in the TOT sample) with a peak of high local velocity dispersion in the central cluster region and low values of local velocity dispersion in external cluster regions (see Fig. 16). We overcame this difficulty by correcting the kinematical estimator. Specifically, we consider the deviation of $\sigma_{V, \text { loc }}$ from the 

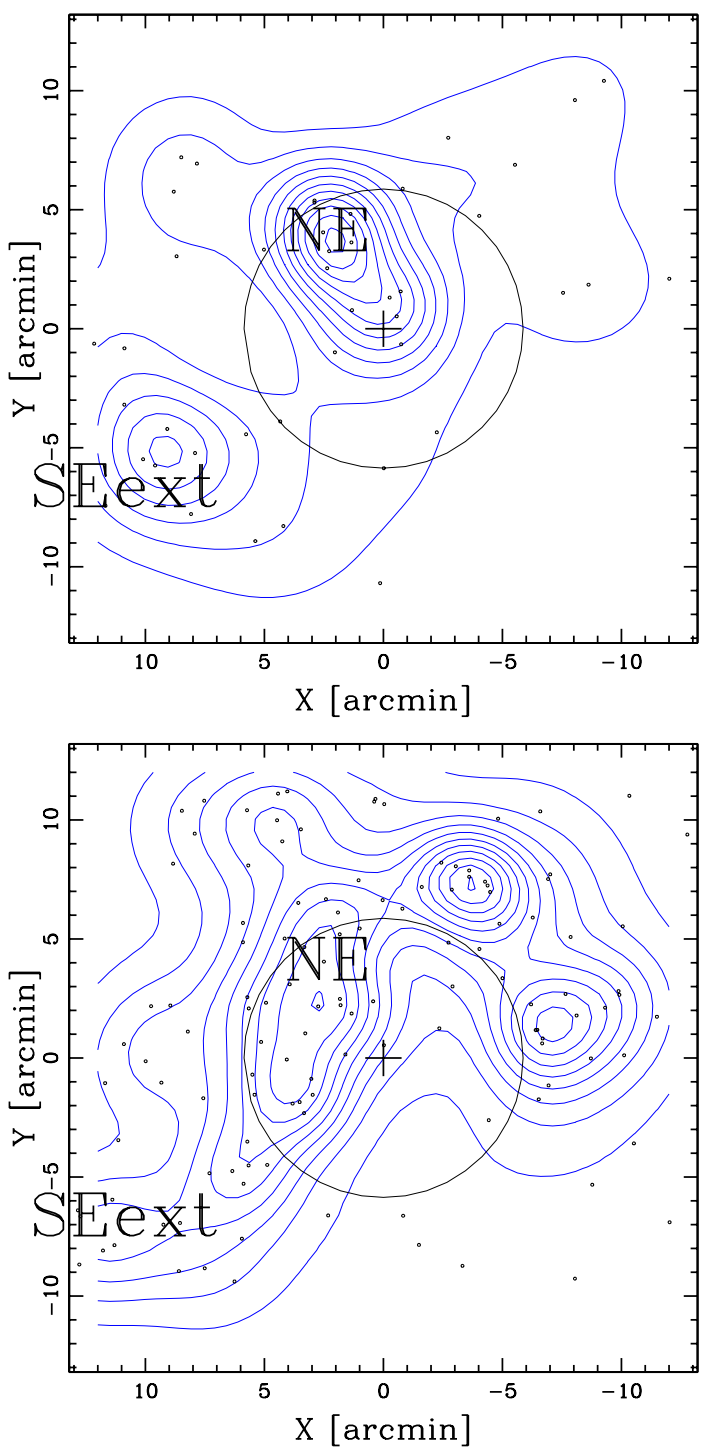

Fig. 12. Spatial distribution and isodensity contour maps of vsELGs (upper panel) and other ELGs (i.e., mELGs+sELGs, lower panel). The position of labels are those in Fig. 11 (lower right panel) to allow an easy comparison with that figure and highlight the concentration of vsELGs in the NE and SEext structures. The $R_{200}$ region is indicated by the circle centered on the BCG.

velocity dispersion expected at the corresponding radius $R$, i.e., $\delta_{i, \mathrm{~s}, \mathrm{corr}}=\left[\left(N_{\mathrm{nn}}+1\right)^{1 / 2} / \sigma_{V}\right] \times\left(\sigma_{V, \text { loc }}-\sigma_{V, R}\right)$. The value of $\sigma_{V, R}$ was obtained by fitting the values of the $\sigma_{V}$-profile in Fig. 7 $\left[\lg \left(\sigma_{V, R} / \mathrm{km} \mathrm{s}^{-1}\right)=3.027-0.224 \times \lg \left(R / h_{70}^{-1} \mathrm{Mpc}\right)\right]$. This correction cancels the spurious signal of substructure in the TOT and other samples (cf. DS $\sigma_{V \text {,corr }}$ and $\mathrm{DS} \sigma_{V}$ values in Table 4 and cf. upper and lower panels in Fig. 16). We note that no significant signal is obtained in the R200 sample, which is by definition less spatially extended, even before the correction.

As for the PAS sample, the $\mathrm{DS} \sigma_{V}$-test substructure signal is not radially symmetric (see Fig. 17) and, as a consequence, does not vanish when applying the correction based on the $\sigma_{V}$ profile. However, the evidence of substructure is always marginal (at the 92-94\% c.1., see Table 4) and few galaxies are related to this 3D substructure. We do not discuss this small feature further.

All the above results are obtained using the classical version of DS-test local groups with $N_{\text {nn }}=10$ neighbors. We also applied the version with $N_{\mathrm{nn}}=20$ for the samples having many

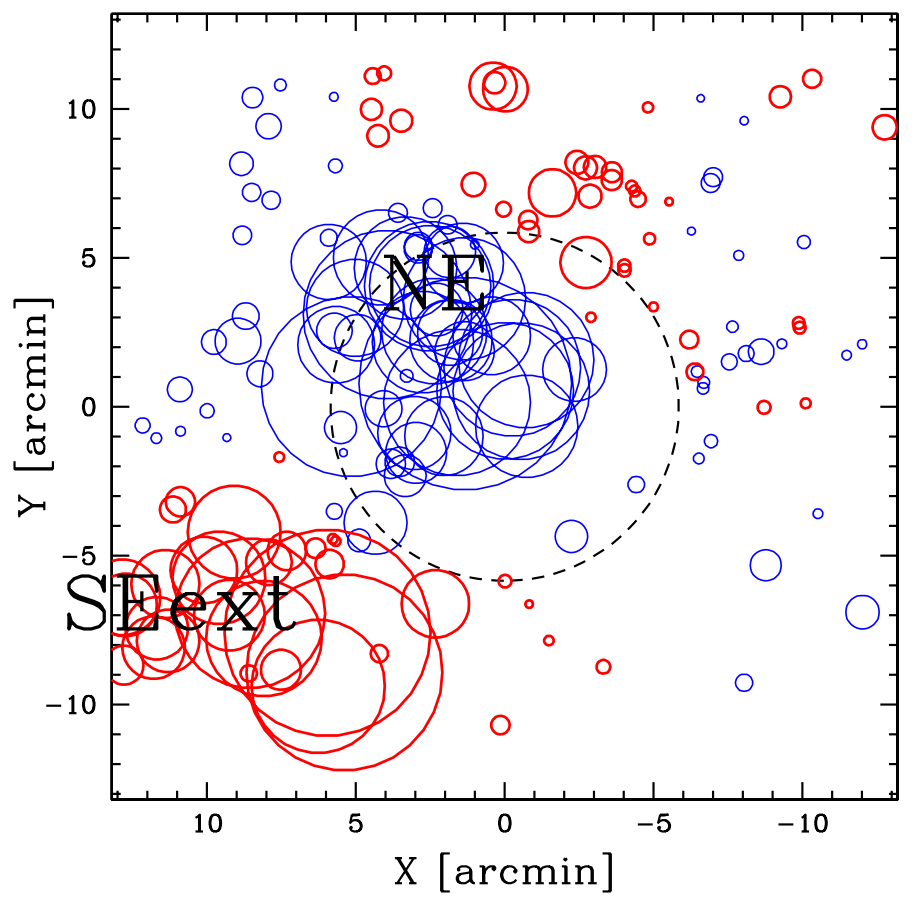

Fig. 13. DS $\langle V\rangle$ bubble-plot for the ELG population. The larger the circle, the larger is the deviation of the local mean velocity from the global mean velocity. Blue and heavy red circles show where the local value of mean velocity is smaller or larger than the global value. Labels indicate the two peaks detected in the 2D analysis (see Table 6 and Fig. 11, lower right panel). The $R_{200}$ region is highlighted by the large black dashed circle centered on the BCG.

members (TOT, R200, PAS, and ELG) with no relevant difference in the results.

\subsection{Substructure analysis as a function of galaxy colors}

We present here the main results of our substructure analysis of MACS1206 by binning the sample on the basis of galaxy colors. We divided the sample into four classes. Starting from the red sequence galaxies, i.e., those with $\left|\left(B-R_{\mathrm{C}}\right)_{\text {diff }}\right| \leq 0.31^{4}$, we defined the red sequence galaxies with positive and negative $\left(B-R_{\mathrm{C}}\right)_{\text {diff }}$, called the RedU sample (72 galaxies) and the RedD sample (133 galaxies), respectively. We then defined a Blue sample, containing 152 galaxies with $\left(B-R_{\mathrm{C}}\right)_{\text {diff }}<-0.5$, and at intermediate colors the Green sample, including 74 galaxies with $-0.5<\left(B-R_{\mathrm{C}}\right)_{\text {diff }}<-0.31$. By repeating the aforementioned analysis per color class, we found no strong evidence of nonGaussianity in the velocity distribution (i.e., always <95\% c.l.), and no evidence of multimodal distributions according to the 1D-DEDICA method. We show the results of the $2 \mathrm{D}$ analysis in Fig. 18. The 2D density maps for Green and Blue galaxies resemble those of wELGs and ELGs in Fig. 11. RedU galaxies are strongly clustered around the cluster center; the WNW peak has a density $\rho_{\mathrm{S}}$ well under the threshold we fixed as being relevant and it is displayed for completeness. RedD galaxies trace the elongated structure of the cluster and, interestingly, confirm the presence of the ESE peak (with 14 objects).

\footnotetext{
4 We adopt a threshold of 0.31 instead of 0.3 because we chose to include the BCG in the red sequence.
} 


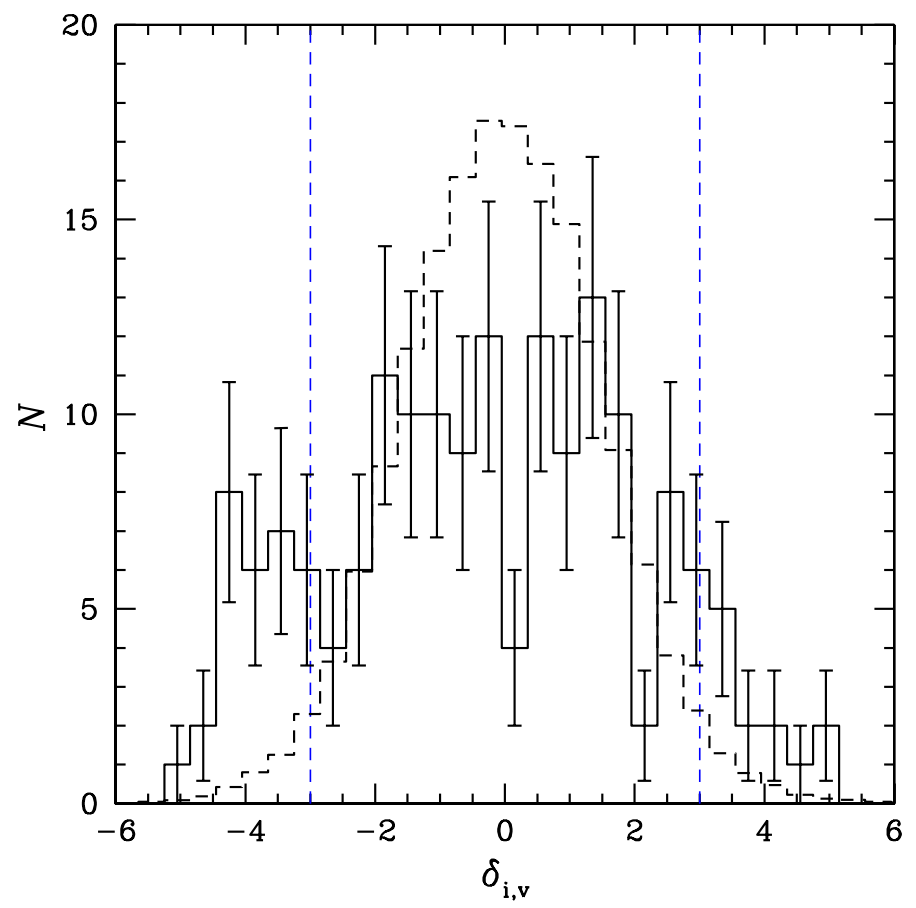

Fig. 14. Distribution of the $\delta_{i, V}$ values for the ELG population. The solid line histogram indicates observed galaxies. The dashed histogram indicates the galaxies of simulated samples, normalized to the observed galaxy number. The blue vertical dashed lines highlight the $\left|\delta_{i, V}\right|>3$ regions where most galaxies are expected to belong to the 3D substructure.

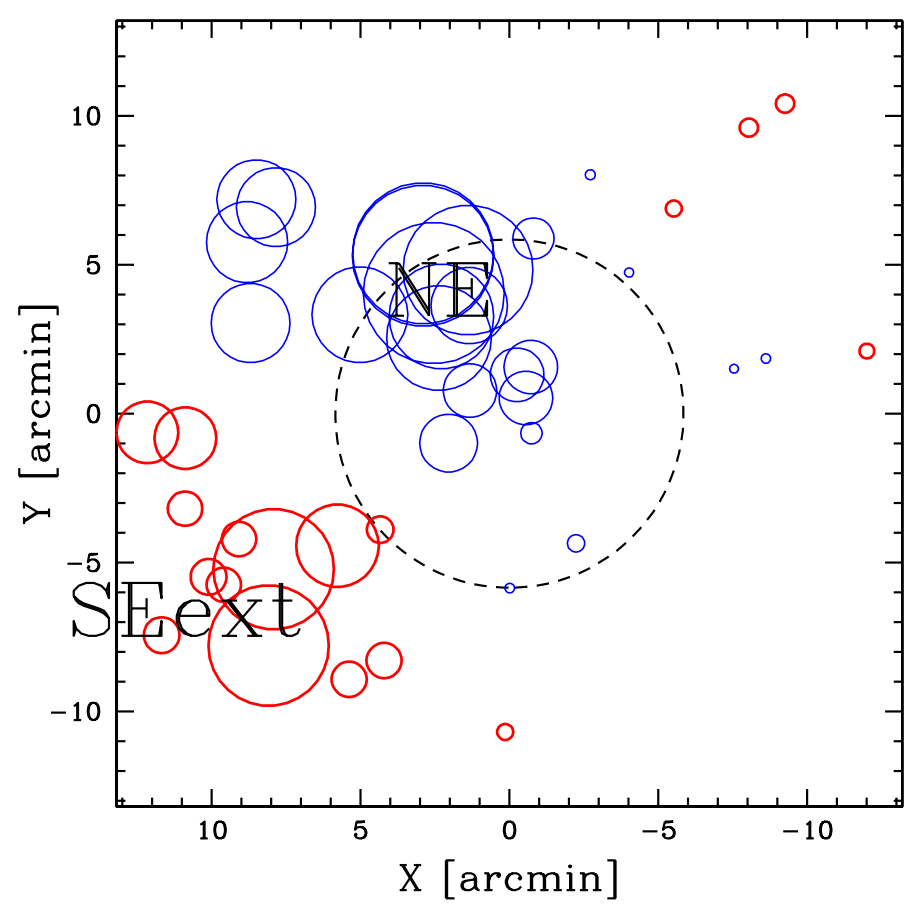

Fig. 15. DS $\langle V\rangle$ bubble-plot as in Fig. 13 but for vsELGs.

\section{General results and discussion}

\subsection{Cluster structure}

The statistical results of the substructure tests applied to the TOT and R200 samples indicate that MACS1206 does not show the presence of large-scale substructure.
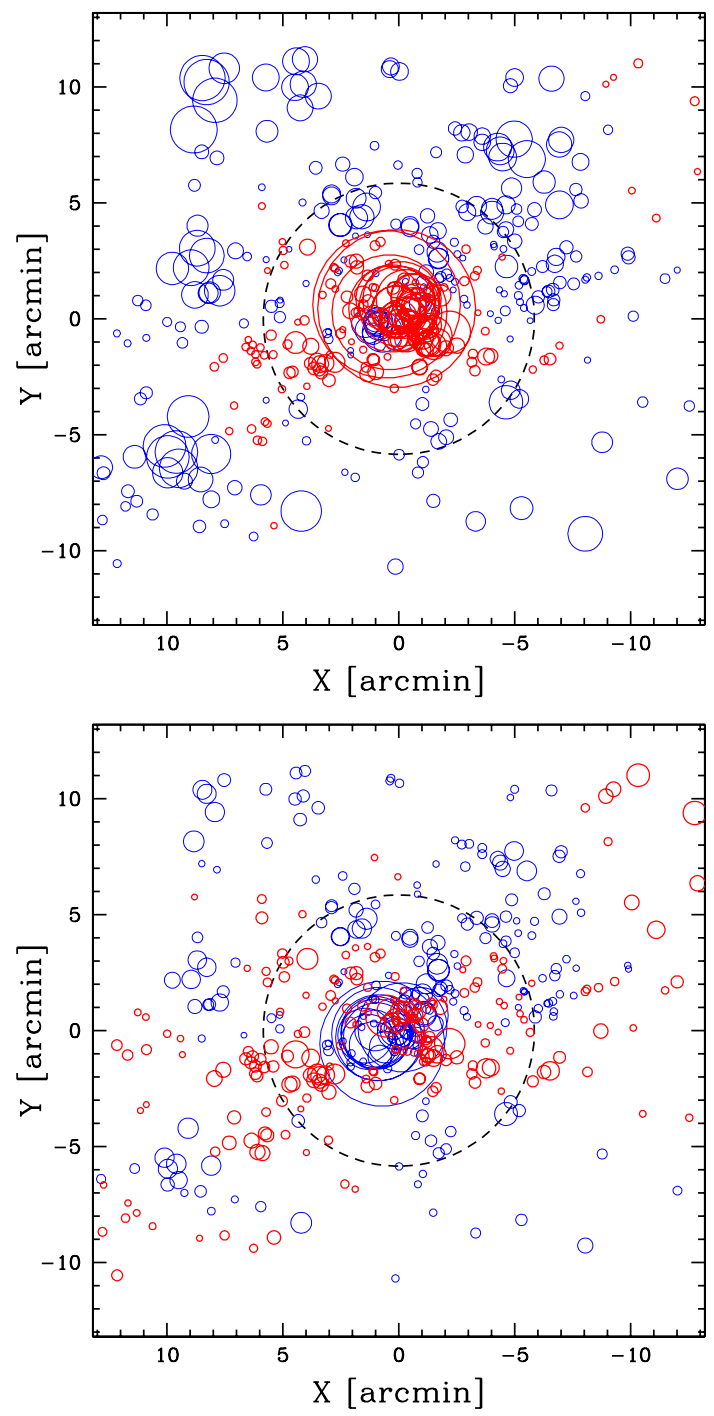

Fig. 16. DS $\sigma_{V}$ bubble-plot in the standard and profile corrected cases for the TOT sample (upper and lower panels). The larger the circle, the larger is the deviation of the local velocity dispersion from the global velocity dispersion. Blue and heavy red circles show where the local value is smaller or larger than the global value. In the upper panel the signal of high local $\sigma_{V}$ in the central region and low local $\sigma_{V}$ in the external region reflect the trend of the $\sigma_{V}$ profile (see Fig. 7, bottom panel) and is no longer significant when applying the suitable correction (lower panel). The $R_{200}$ region is indicated by the dashed circle centered on the BCG.

The 2D elongation is the most important large-scale feature in the cluster structure. In the plane of the sky the cluster structure appears clearly elongated along the WNW-ESE direction (see Fig. 10). We obtained PA $=102_{-8}^{+7}$ and $117_{-4}^{+3}$ deg for galaxies in the R200 and TOT samples, respectively. This orientation matches that of the mass distribution from strong gravitational lensing (see Fig. 1 in Zitrin et al. 2012), BCG shape, X-ray isophotes, and the mass distribution from weak gravitational lensing (PA in the range 105-119 ${ }^{\circ}$, Table 6 in Umetsu et al. 2012), the asymmetric distribution of the intracluster light (PA of BCG and ICL in the range 101-109 ${ }^{\circ}$, Presotto et al. 2014). Our novel result is that there is neither evidence for a velocity gradient along the WNW-ESE direction nor for a velocity difference between the main system and most subclumps. This suggests that the main MACS1206 structure is lying in the plane of the sky. 


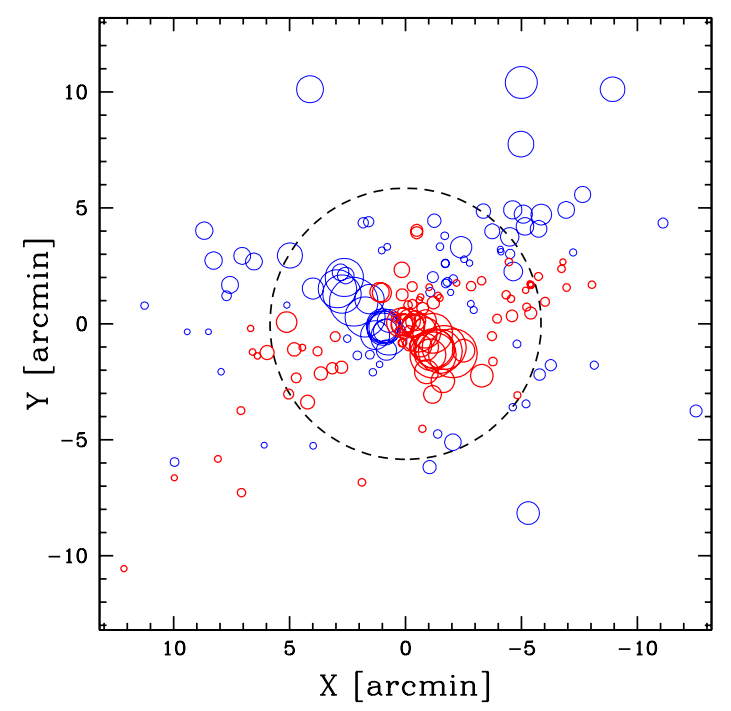

Fig. 17. DS $\sigma_{V}$ bubble-plot as in Fig. 16 (standard case), but for the PAS population.
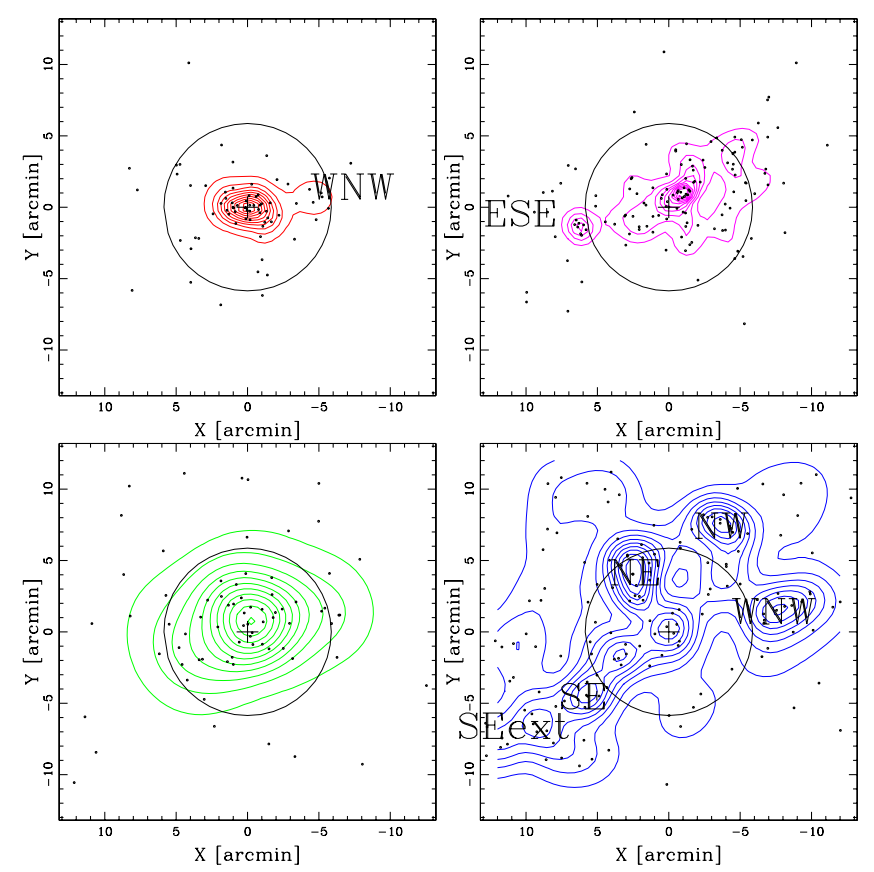

Fig. 18. Spatial distributions and isodensity contour maps for the four color classes: RedU (upper left panel), RedD (upper right panel), Green (lower left panel), and Blue (lower right panel) populations. Labels are the same as in Fig. 11.

As for the interpretation, it is well known that cluster's dynamical activity is strongly correlated with the tendency of clusters to be elongated and aligned with the nearby clusters (Plionis $\&$ Basilakos 2002) because of the anisotropic merging along the LSS filaments as also shown by the analysis of simulations (e.g., Basilakos et al. 2006; Cohen et al. 2014). However, it is also known that the final distribution after violent relaxation may be aspherical (Aarseth \& Binney 1978; White 1996). Thus, in principle, the elongation of MACS1206 is not in contrast with the lack of statistical evidence for the presence of important substructure.

An interesting global parameter is the value of the ratio between the energy per unit mass of galaxies to that of ICM as parametrized with $\beta_{\text {spec }}=\sigma_{V}^{2} /\left(k T_{\mathrm{X}} / \mu m_{\mathrm{p}}\right)$, where $T_{\mathrm{X}}$ is the X-ray temperature, $\mu=0.58$ is the mean molecular weight and $m_{\mathrm{p}}$ the proton mass. The value $\beta_{\text {spec }}=1$ indicates the densityenergy equipartition between ICM and galaxies. The mean $\beta_{\text {spec }}$ value observed for massive clusters is consistent with unity both in nearby (Girardi et al. 1996, 1998) and distant systems out to $z \sim 0.4$ (Mushotzky \& Scharf 2004), while large deviations may be related to important cluster merging phenomena (e.g., Ishizaka 1997). In the case of MACS1206, the value of $k T_{\mathrm{X}}=10.8 \pm 0.6 \mathrm{keV}$ has been obtained by Postman et al. (2012) using Chandra data within a radius of $0.714 h_{70}^{-1} \mathrm{Mpc}$ and excluding the very central cluster region. Using our estimate of $\sigma_{V}$ within the same radius, we obtained $\beta_{\mathrm{spec}}=0.96 \pm 0.14$, in agreement with the scenario of density-energy equipartition (see also Fig. 7, bottom panel).

At small scale, the analysis of the $2 \mathrm{D}$ galaxy distribution shows three secondary peaks with low values of relative density $\rho_{\mathrm{S}} \lesssim 0.2$ with respect to the main cluster peak (WNW, SEint, ESE in the TOT sample, see Table 6). The ESE and WNW peaks have already been detected as significant overdensities by Lemze et al. (2013), who have used a different sample and method. In the R200 sample, the SEint density peak is the only one detected, leading to a $12 \%$ of cluster members ( $8 \%$ in luminosity) related to the substructure. Among clusters with no major substructure, the typical fraction of galaxies in substructure is $\sim 10 \%$ (e.g., Girardi et al. 1997; Guennou et al. 2014). In the TOT sample, which extends out to $R \sim 2 R_{200}$ we formally assigned $52 \%$ of the galaxies to substructure. However, we stress that the precise number of objects assigned to the WNW clump is largely uncertain owing to possible border effects. The WNW clump contains galaxies out to the western boundary of the sampled region. We checked this point extending the size of our sample out to $\sim 4 R_{200}$ with the addition of a random background field. The addition of a field equal to $5 \%$ of the TOT sample does not change the value of the relative density $\rho_{\mathrm{S} \text {,WNW }}$ of the WNW peak, but reduces the number of the assigned WNW members. The fraction of WNW members in the TOT sample changes from WNW/TOT $~ 23 \%$ to $\mathrm{WNW} / \mathrm{TOT} \sim 10 \%$ leading to an important reduction in the estimated fraction of galaxies in substructures. In any case, our result agrees with substructure being more important in the external cluster regions (e.g., West \& Bothun 1990).

\subsection{Tracing a past phase of accretion}

Both the PAS and HDSr populations trace a cluster structure elongated along the WNW-ESE direction suggesting that this is the direction of the main accretion filament during the cluster formation. Our interpretation is that HDSr galaxies represent the galaxies more affected by the last important phase of accretion, likely a past merger or mergers along the WNW-ESE direction. In fact, the HDSr spectral features reflect the evolution of galaxies having undergone starburst or star-forming activity, which has been suppressed by some physical process, possibly related to the high-density environment, and observed 1-2 Gyr later (see, e.g., M04). This time is comparable with the crossing time of the present cluster $\left(R_{200} / \sigma_{V} \sim 1.8 \mathrm{Gyr}\right)$ indicating that the supposed merger(s) are in an advanced phase. This also agrees with the fact that we do not see relevant features of this past dynamical activity, since the populations of the intervening subclusters have had time to mix. In principle, we cannot exclude a phase of violent relaxation, since its time scale is comparable to the dynamical time. A time estimate of 1-2 Gyr is also consistent with the elongation of the X-ray isophotes which is a long-lived phenomenon (e.g., Roettiger et al. 1996) and the asymmetry of the ICL, whose surviving time can be comparable with one crossing time (Rudick et al. 2009). 


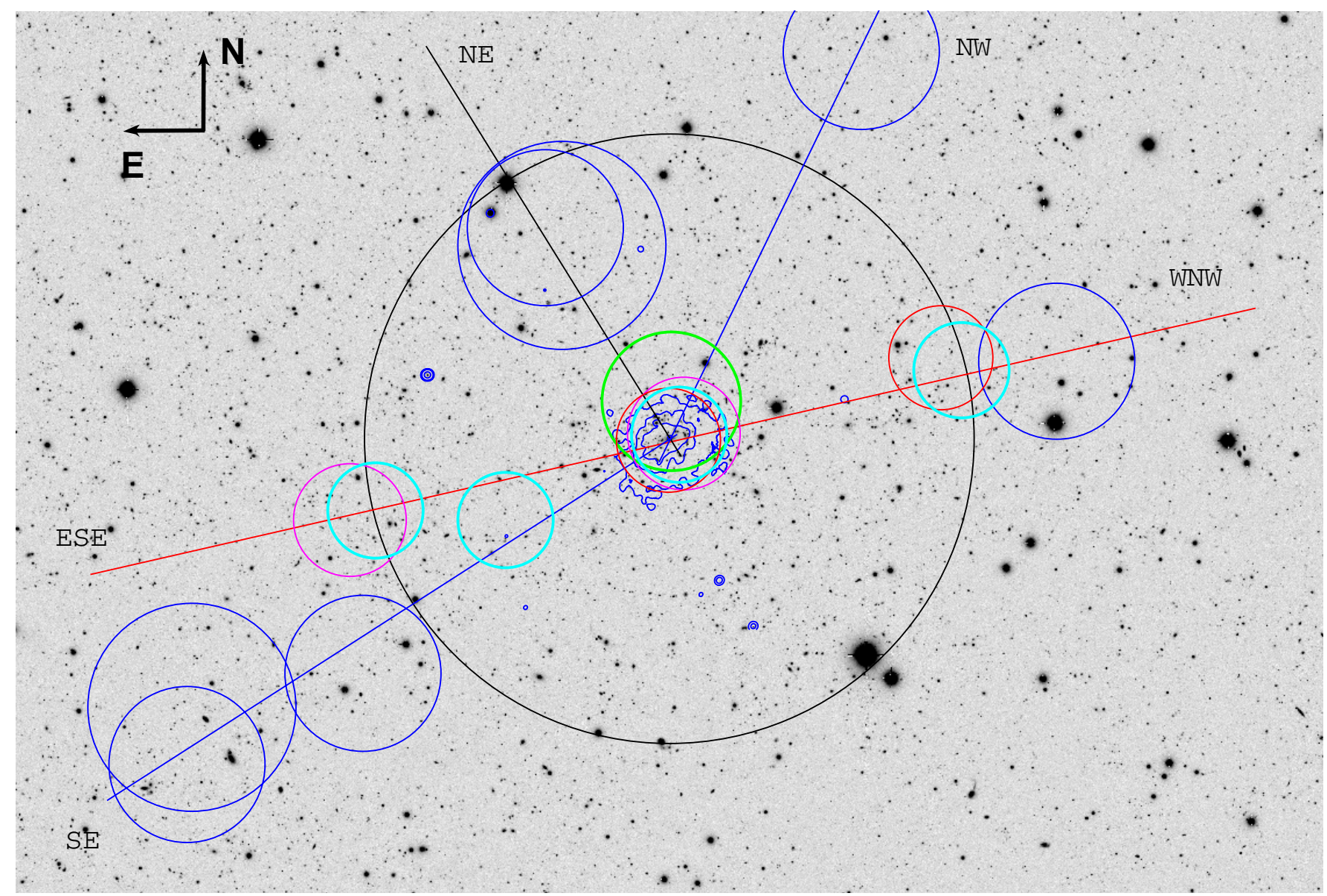

Fig. 19. Subaru $R_{\mathrm{C}}$ image of MACS1206 with superimposed features summarizing the results of the cluster structure analysis. Circles, in increasing size order, indicate the density peaks detected in the 2D galaxy distribution of TOT (cyan), PAS (red), HDSr (magenta), wELG (green), ELG and vsELG (blue) samples (see Table 6 and Fig. 11); the NE and SEext clumps being also detected through our 3D analysis. The lines indicate the likely directions of cluster accretion. Blue contours are the smoothed contour levels of the X-ray surface brightness derived from Chandra archival data (in the 0.5-2 keV energy range; see also Ebeling et al. 2009). The large black circle encloses the $R_{200}=2 h_{70}^{-1} \mathrm{Mpc} \sim 5.9^{\prime}$ region.

The observational evidence in favor of a past merger along the WNW-ESE direction is the presence of a secondary dense peak in the distribution of HDSr galaxies (ESE peak), confirmed in the distribution of RedD galaxies, too (see Figs. 11 and 18, upper right panels). The mean values of $E W(\mathrm{H} \delta)$ of the RedU and RedD populations $(0.19 \pm 0.35 \AA$ and $1.10 \pm 0.24 \AA)$ are only $2 \sigma$ different, but in the direction of reinforcing our idea that the ESE peak is only traced by the younger population (RedD). The HDSr ESE peak might be the remnant of a galaxy system, now merged to form MACS1206. However, it is formed only of faint galaxies $\left(R_{\mathrm{C}}>21.5 \mathrm{mag}\right)$, lacking a dominant galaxy which would have supported its identification with the remnant core of an important subcluster. Another point in favor of a past merger along the WNW-ESE direction is the presence of a bright galaxy (at RA $=12^{\mathrm{h}} 06^{\mathrm{m}} 15^{\mathrm{s}} 66$, Dec $=-08^{\circ} 48^{\prime} 21.8^{\prime \prime} \quad(\mathrm{J} 2000)$; also G2 in Grillo et al. 2014b), which is the second brightest galaxy within $0.5 h_{70}^{-1} \mathrm{Mpc}$ from the cluster center, and which lies at the extremity of the asymmetric ICL distribution, and seems to have strongly suffered from the interaction with the BCG and/or the central cluster potential (see Fig. 6 of Presotto et al. 2014). This galaxy is also just at the border of our inclusion in the HDSr sample $\left(E W\left(\mathrm{H}_{\delta}\right)=3 \pm 1 \AA\right)$. A third point is the WNW overdensity in the spatial distribution of PASs, although the presence of a close overdensity of ELGs makes the interpretation difficult.

To understand the physical mechanisms generating HDSr galaxies, we note that, at large scale, their spatial distribution follows that of PAS galaxies. This agrees with the findings of Dressler et al. (2013), who also suggested an evolutionary path of the type $\mathrm{PAS} \Rightarrow \mathrm{PSB} \Rightarrow \mathrm{PAS}$. However, we cannot completely agree with their scenario since our morphological analysis based on Sérsic indices shows that HDSr galaxies are intermediate between PAS and ELG galaxies (see Fig. 3). The intermediate morphology suggests that HDSr galaxies are the result of two mixed populations or that the physical process involved in their transformation works on both their gas content and morphology. The second interpretation favors a scenario of merger or tidal interaction rather than a gas shocking or stripping. In particular, the dense HDSr ESE concentration could be explained by a mechanism similar to that suggested by Struck (2006). According to this mechanism, the gravitational pull of the cluster core on a small group falling through the core will cause the group to shrink, increasing its density by an order of magnitude, and as a result increasing the galaxy merger rate.

\subsection{Recent and ongoing galaxy infall}

Thanks to the large spatial coverage of the spectroscopic sample, the ELG population can be studied in detail. We identify several clumps (see Fig. 11, right lower panel). These galaxy clumps are very loose and should be interpreted as overdensities in the LSS filaments accreted to the cluster rather than gravitationally bound, isolated groups. These structures are generally external 
to $R_{200}$ with the exception of the NE clump which, being characterized by a $\Delta V_{\mathrm{NE}, \mathrm{rf}} \sim-550 \mathrm{~km} \mathrm{~s}^{-1}$, might be seen there because of projection effects. The ELG clumps we detect are likely the cluster building blocks during the ongoing galaxy infall.

$N$-body simulations by Balogh et al. (2000) and Moore et al. (2004) follow the so-called backsplash or overshoot of galaxies that have passed through the cluster and joined the virialized systems: both these studies identify the zone between 1 and $2 R_{200}$ as the overlap region where roughly half the galaxies are members of the cluster and half are infalling. Since individual ELG galaxy clumps are still detectable, we expect that their members are likely infalling rather than backsplashing. Considering the region between 1 and $2 R_{200}$, the ratio between galaxies that belong to the ELG structures and the whole population is $93 / 161 \sim 0.6$. Our result suggests that $60 \%$ of galaxies have never passed through the cluster in rough agreement with the $50 \%$ predicted by simulations by Balogh et al. (2000) and Moore et al. (2004).

When analyzing the geometry of the infall, the ELG clumps provide a few preferred directions, traced in Fig. 19 roughly connecting the clumps and the cluster center. Figure 19 shows how both the WNW-ESE and NW-SE directions are somewhat matched in the elongation of X-ray isophotes, which are tilted going from the internal to the external regions. The net result of the secondary infall is a somewhat rounder assembling than that due to the past WNW-ESE accretion as suggested by the different ellipticity between PAS and ELG galaxies (see Fig. 9 and Table 5).

The galaxies of the vsELG class are spatially segregated with respect to those of $\mathrm{mELG}+\mathrm{sELG}$ classes, clustering in the NE and SEext clumps. Figure 20 shows the values of stellar mass and SFR that Annunziatella et al. (2014) computed for cluster member galaxies. Comparing vsELGs and other ELGs in the $10^{9} \lesssim M_{*} / M_{*, \odot}<10^{10}$ range, we note that the specific SFR of vsELGs is higher, particularly at low stellar masses. We interpret the observed spatial segregation as the result of the same physical process simultaneously acting in most galaxies within the parent clump, which is enhancing SF in the NE and SEext clumps, or, alternatively, quenching SF in other clumps. In order to understand whether this process is connected to the cluster or rather to a pre-infalling environment, we analyzed the relative fraction of vsELGs in the cluster and in the field. To this end, we selected a representative set of 776 field galaxies as non-member galaxies in the $0.2<z<0.7$ redshift range, with spectral classification. We compared the fraction of vsELGs with respect to all ELGs. We obtained that the vsELG/ELG ratio in the cluster, $43 / 168$ (26\%), is completely consistent with the one in the field, $160 / 602(27 \%)$. This does not support a scenario where cluster environment is relevant in determining the vsELG/ELG ratio.

In the context of possible infalling galaxy structures, we stress that our study focuses on galaxies belonging to the main cluster peak in the redshift distribution, and does not consider foreground or background redshifts peaks. In the study of Young et al. (2014), a few galaxies in a foreground redshift peak are related to the presence of a significant interesting SZE feature, and interpreted as a foreground or infalling group.

\subsection{Intermediate galaxy populations}

Here we discuss the two intermediate populations, wELG and HDSb, which according to our interpretation represent the fate of recently in-fallen galaxies. The HDSb galaxies in our study are comparable to the PSBs studied by Muzzin et al. (2014),

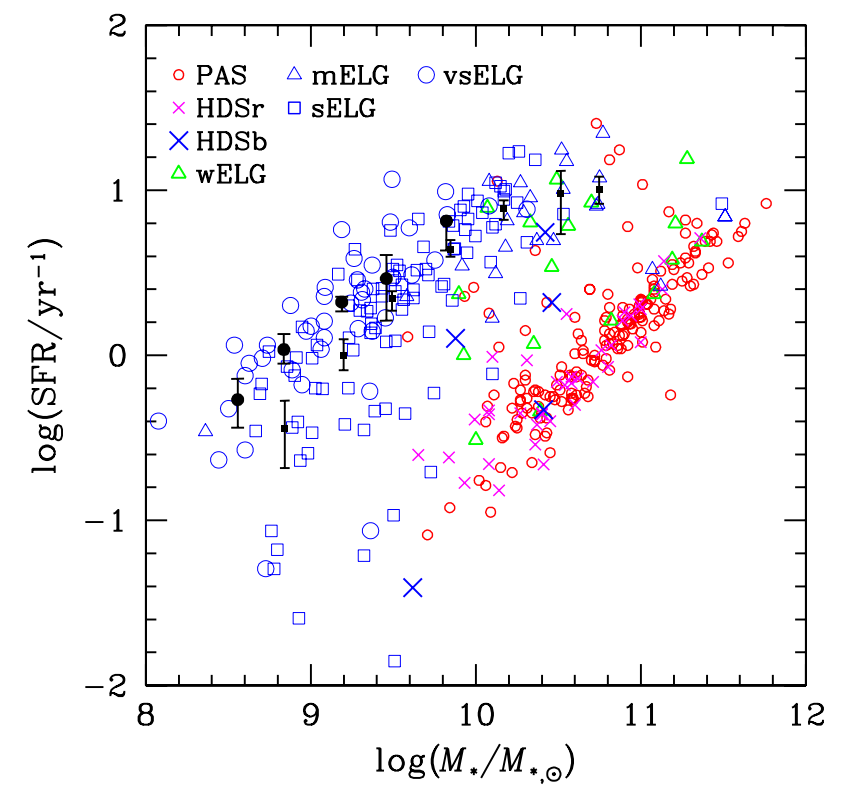

Fig. 20. SFR vs. stellar mass of cluster galaxies. Individual values are those computed by Annunziatella et al. (2014) and are shown here for different spectral classes. Black solid circles and small squares indicate biweight means with bootstrap errors for vsELGs and mELGs+sELGs considering three bins per order of magnitude in stellar mass.

which have very strong $E W(\mathrm{H} \delta)(E W(\mathrm{H} \delta)>5 \AA$ for the average stacked spectrum) and are defined with $D_{n}(4000)<1.45$, i.e., are blue PSBs (see Fig. 2 of M04). Figure 21 shows that four of the five HDSb galaxies in our sample are located within or close to the same ring-like region in the projected phase space indicated by Muzzin et al. (2014, see their Fig. 1). In that study PSBs are explained with a rapid quenching of the order of $\lesssim 0.5 \mathrm{Gyr}$ after making their first passage of $R \sim 0.5 R_{200}$. The same time scale of $\sim 0.5$ Gyr is invoked by M04 after the interruption of the SF in the HDSb galaxies. In MACS1206, the five HDSb galaxies are elongated towards the SE direction, approximately in the NW-SE direction of the putative accretion flow (cf. Figs. 4 and 19). The wELG population appears distinct from the ELG population, since wELGs populate inner cluster regions, and from the PAS and HDSr populations since wELGs do not follow the WNW-ESE elongation. Our interpretation is that wELGs are associated with a more advanced phase in the ELG infalling process.

\section{Summary, conclusions, and perspectives}

To elucidate the scenario and physical mechanism leading to the assembly of galaxy clusters, and specifically the transformation of star-forming field galaxies to passive cluster galaxies, we present a detailed spectrophotometric study of the massive cluster MACS1206 at $z=0.44$ as part of the CLASH survey. Our analysis is based on an extensive spectroscopic dataset of 1920 galaxies over a field of $640 \mathrm{arcmin}^{2}$ around MACS1206, which was the first observing campaign conducted with VIMOS at the VLT as part of the "CLASH-VLT" ESO Large Programme including 13 CLASH clusters in the southern sky. The dataset is supplemented with multiband photometry based on Subaru Suprime-Cam high-quality imaging. We combine galaxy velocities and positions to select 445 cluster galaxies based on kinematic information alone. Using spectral absorption and emission features, as well as galaxy colors, we classify 412 galaxies in seven spectral classes ranging from purely passive galaxies to 


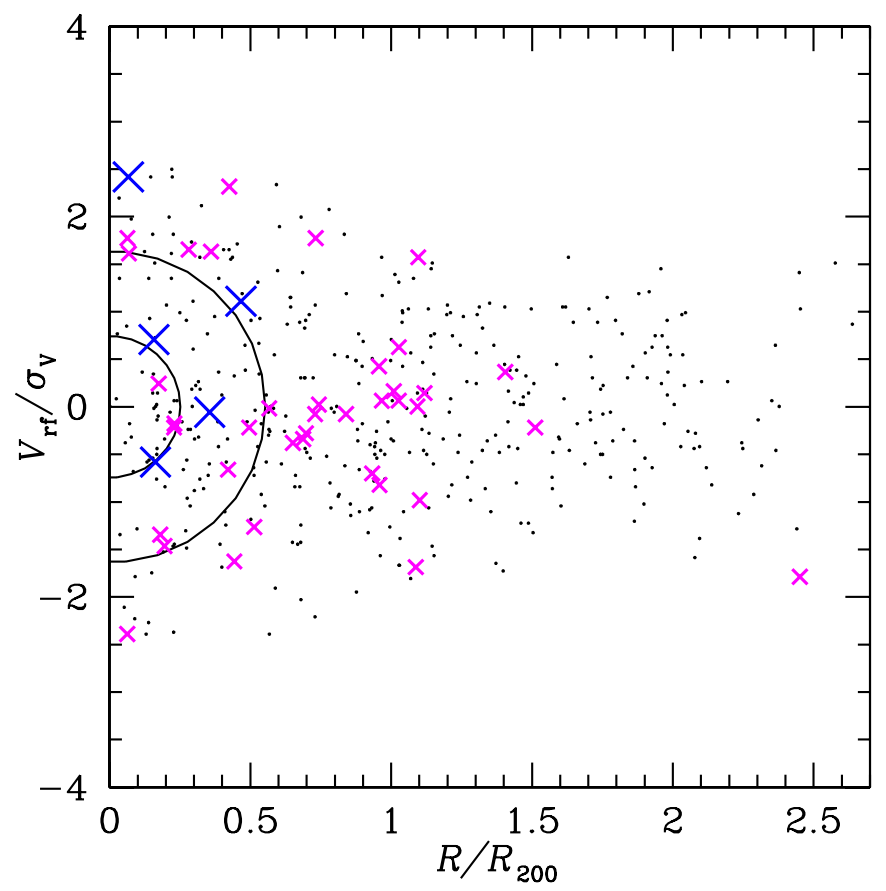

Fig. 21. Projected phase space as in Fig. 7, top panel, but with normalized units in position and velocity axes. Small magenta crosses and large blue crosses indicate HDSr and HDSb galaxies. Four of the five HDSb galaxies are located within or close to the same ring-like region traced by the cluster PSBs analyzed by Muzzin et al. (2014); see text for details.

those with strong $\mathrm{H} \delta$ absorption (red and blue), and emission line galaxies (four classes from weak to very strong lines). We then analyze the cluster structure and distribution of these different galaxy populations in projected phase-space out to a radius $\sim 2 R_{200}\left(4 h_{70}^{-1} \mathrm{Mpc}\right)$. Our specific results can be summarized as follows:

- MACS1206 appears as a large-scale relaxed system, particularly within $R_{200}$, with a few, low overdensities detected in the projected galaxy distribution (relative density $\$ 20 \%$ ). The whole galaxy population does not show velocity gradients or evidence of significant deviations in local mean velocities. The main large-scale feature is the elongation along the WNW-ESE direction $\left(\mathrm{PA} \sim 117^{\circ}\right.$ ).

- Passive galaxies and red, strong $\mathrm{H} \delta$ galaxies trace the cluster center and the WNW-ESE elongated structure. The latter also cluster around a secondary, very dense peak $2 h_{70}^{-1} \mathrm{Mpc}$ at ESE.

- The emission line galaxies are mostly located in several loose structures, mostly outside $R_{200}$. Two of these structures are also detected in the $3 \mathrm{D}$ projected phase space having LOS rest-frame velocities $\Delta V_{\mathrm{rf}, \mathrm{NE}} \sim-550 \mathrm{~km} \mathrm{~s}^{-1}$ and $\Delta V_{\text {rf,SEext }} \sim+550 \mathrm{~km} \mathrm{~s}^{-1}$.

- The clumps of emission line galaxies trace several directions, with the net result of a more symmetrical distribution than that traced by early-type galaxies (the difference in ellipticity is a factor of 1.5-2).

By studying the spatial and velocity distributions of galaxy populations of different spectral types, we can piece together a likely scenario for the galaxy assembly history of this massive system. The oldest and main component of MACS1206, made of early-type galaxies, clearly traces the main phase of cluster formation. However, a more recent anisotropic accretion phase that occurred 1-2 Gyr before the epoch of observation is apparent in the distribution of red strong $\mathrm{H} \delta$ galaxies along the prominent WNW-ESE filament, including a strong overdensity of these evolved post-SF galaxies (ESE peak), possibly the remnant of an accreted group. In addition, we identified a secondary prominent young component in the external regions of MACS1206 populated by late-type galaxies, which trace the ongoing infall.

Our study shows that large spectroscopic samples of cluster galaxies extending to $\gtrsim 2 R_{200}$ allow us to link the cluster substructure with distinct galaxy populations, whose spectral types can be used to age-date their underlying stellar populations and SF history. This ultimately allows us to reconstruct the evolution of galaxies and their parent cluster, thus going beyond the simplistic concept of radial accretion and following cluster formation through their building blocks. We plan to extend this analysis to other clusters in the CLASH-VLT survey, which include systems of different dynamical states and over an interesting redshift range. A more refined spectral classification will also be possible in clusters in this program, which include $\sim 7000 \mathrm{spec}-$ troscopic members.

Acknowledgements. We thank the referee for invaluable comments. This research is partly supported by the MIUR PRIN2010-2011 (J91J12000450001) and PRIN INAF 2014. I.B. acknowledges funding from the European Union Seventh Framework Programme (FP7/2007-2013) under grant agreement n. 267251 "Astronomy Fellowships in Italy" (astroFit). The Dark Cosmology Centre is funded by the DNRF. B.S. and V.P. acknowledge a grant from "Consorzio per la Fisica - Trieste". R.D. gratefully acknowledges the support provided by the BASAL Center for Astrophysics and Associated Technologies (CATA), and by FONDECYT grant N. 1130528. A.F. acknowledges support by INAF through VIPERS grants PRIN 2008 and PRIN 2010. Support for A.Z. was provided by NASA through Hubble Fellowship grant \#HST-HF2-51334.001-A awarded by STScI. The research is based in part on data collected at Subaru Telescope, which is operated by the National Astronomical Observatory of Japan, and obtained from the SMOKA, which is operated by the Astronomy Data Center, National Astronomical Observatory of Japan.

\section{References}

Aarseth, S. J., \& Binney, J. 1978, MNRAS, 185, 227

Abraham, R. G., Smecker-Hane, T. A., Hutchings, J. B., et al. 1996, ApJ, 471, 694

Adami, C., Biviano, A., \& Mazure, A. 1998, A\&A, 331, 439

Annunziatella, M. Biviano, A., Mercurio, A., et al. 2014, A\&A, 571, A80

Athreya, R. M., Mellier, Y., van Waerbeke, L., et al. 2002, A\&A, 384, 743

Baier, F. W., \& Ziener, R. 1977, Astron. Nachr., 298, 87

Balogh, M. L., Morris, S. L., Yee, H. K. C., Carlberg, R.G., \& Ellingson, E. 1999, ApJ, 527, 54

Balogh, M. L., Navarro, J. F., \& Morris, S. L. 2000, ApJ, 540, 113

Barden, M., Rix, H.-W., Somerville, R. S., et al. 2005, ApJ, 635, 959

Barden, M., Häussler, B., Peng, C. Y., McIntosh, D. H., \& Guo, Y. 2012, MNRAS, 422, 449

Barger, A., Aragon-Salamanca, A., Ellis, R., et al. 1996, MNRAS, 279, 1

Barrena, R., Girardi, M., Boschin, W., et al. 2011, A\&A, 529, A128

Basilakos, S., Plionis, M., Yepes, G., Gottlöber, S., \& Turchaninov, V. 2006 MNRAS, 365, 539

Beers, T. C., Flynn, K., \& Gebhardt, K. 1990, AJ, 100, 32

Bekki, K. 1999, ApJ, 510, L15

Bekki, K., Owers, M. S., \& Couch, W. J. 2010, ApJ, 718, L27

Bird, C. M., \& Beers, T. C. 1993, AJ, 105, 1596

Biviano, A., Girardi, M., Giuricin, G., Mardirossian, F., \& Mezzetti, M. 1992, ApJ, 396, 35

Biviano, A., Katgert, P., Mazure, A., et al. 1997, A\&A, 321, 84

Biviano, A., Katgert, P., Thomas, T., \& Adami, C. 2002, A\&A, 387, 8

Biviano, A., Rosati, P., Balestra, I., et al. 2013, A\&A, 558, A1 (B13)

Böhringer, H., Pratt, Schuecker, P., Guzzo, L., et al. 2004, A\&A, 425, 367

Böhringer, H., Pratt, G. W., Arnaud, M., et al. 2010, A\&A, 514, A32

Buote, D. A. 2002, in Merging Processes in Galaxy Clusters, eds. L. Feretti, I. M. Gioia, \& G. Giovannini (The Netherlands: Kluwer Ac. Pub.), Astrophys. Space Sci. Lib., 272, 79 
Butcher, H., \& Oemler, A., Jr. 1984, ApJ, 285, 426

Caldwell, N., \& Rose, J. A. 1997, AJ, 113, 492

Carter, D., \& Metcalfe, J. 1980, MNRAS, 191, 325

Cohen, S. A., Hickox, R. C., Wegner, G. A., Einasto, M., \& Vennik, J. 2014, ApJ, 783, 136

Colberg, J. M., White, S. D. M., Macfarland, T. J., et al. 1998, in Wide Field Surveys in Cosmology (Edition Frontiers), 247

Colberg, J. M., White, S. D. M., Jenkins, A., \& Pearce, F. R. 1999, MNRAS, 308, 593

Colless, M., \& Dunn, A. 1996, ApJ, 458, 435

Couch, W. J., \& Sharples, R. M. 1987, MNRAS, 229, 423

Czoske, O., Moore, B., Kneib, J.-P., \& Soucail, G. 2002, A\&A, 386, 31

Dahle, H., Kaiser, N., Irgens, R. J., Lilje, P. B., \& Maddox, S. J. 2002, ApJS, 139,313

Danese, L., De Zotti, C., \& di Tullio, G. 1980, A\&A, 82, 322

Demarco, R., Rosati, P., Lidman, C., et al. 2007, ApJ, 663, 164

den Hartog, R., \& Katgert, P. 1996, MNRAS, 279, 349

Dietrich, J. P., Werner, N., Clowe, D., et al. 2012, Nature, 487, 202

Donahue, M., Voit, G. M., Gioia, I. M., et al. 1998, ApJ, 502, 550

Dressler, A. 1980, ApJ, 236, 351

Dressler, A., \& Gunn, J. E. 1983, ApJ, 270, 7

Dressler, A., \& Shectman, S. A. 1988, AJ, 95, 985

Dressler, A., Oemler, A. Jr., Couch, W. J., et al. 1997, ApJ, 490, 577

Dressler, A., Smail, I., Poogianti, B. M., et al. 1999, ApJ, 122, 51

Dressler, A., Rigby, J, Oemler, A. Jr., et al. 2009, ApJ, 693, 140

Dressler, A., Oemler, A. Jr., Poggianti, B. M., et al. 2013, ApJ, 770, 62

Ebeling, H., Ma, C. J., Kneib, J.-P. Kneib, et al. 2009, MNRAS, 395, 1213

Fadda, D., Girardi, M., Giuricin, G., Mardirossian, F., \& Mezzetti, M. 1996, ApJ, 473, 670

Fadda, D., Biviano, A., Marleau, F. R., Storrie-Lombardi, L. J., \& Durret, F. 2008, ApJ, 672, L9

Fasano, G., \& Franceschini, A. 1987, MNRAS, 225, 155

Fassbender, R., Böhringer, H., Nastasi, A., et al. 2011, New J. Phys., 13, 125014

Feretti, L., Gioia, I. M., \& Giovannini G. 2002b, Merging Processes in Galaxy Clusters, (The Netherlands: Kluwer Academic Publisher), Astrophys. Space Sci. Lib., 272

Ferrari, C., Benoist, C., Maurogordato, S., Cappi, A., \& Slezak, E. 2005, A\&A, 430, 19

Fisher, D. B., \& Drory, N. 2008, AJ, 136, 773

Fritz, A., Ziegler, B. L., Bower, R. G., Smail, I., \& Davies, R. L. 2005, MNRAS, 358,233

Fujita, Y., Takizawa, M., Nagashima, M., \& Enoki, M. 1999, PASJ, 51, L1

Gebhardt, K., \& Beers, T. C. 1991, ApJ, 383, 72

Geller, M. J., \& Beers, T. C. 1982, PASP, 94, 421

Gerken, B., Ziegler, B., Balogh, M., et al. 2004, A\&A, 421, 59

Gioia, I. M., Henry, J. P., Mullis, C. R., Ebeling, H., \& Wolter, A. 1999, AJ, 117, 2608

Girardi, M., \& Biviano, A. 2002, in Merging Processes in Galaxy Clusters, eds. L. Feretti, I. M. Gioia, \& G. Giovannini (The Netherlands: Kluwer Ac. Pub.), Astrophys. Space Sci. Lib., 272, 39

Girardi, M., Fadda, D., Giuricin, G., et al. 1996, ApJ, 457, 61

Girardi, M., Escalera, E., Fadda, D., et al. 1997, ApJ, 482, 11

Girardi, M., Giuricin, G., Mardirossian, F., Mezzetti, M., \& Boschin, W. 1998, ApJ, 505, 74

Girardi, M., Boschin, W., \& Barrena, R. 2006, A\&A, 455, 45

Girardi, M., Bardelli, S., Barrena, R., et al. 2011, A\&A, 536, A89

Gómez, P. L., Nichol, R. C., Miller, C. J., et al. 2003, ApJ, 584, 210

Grillo, C., Suyu, S. H., Rosati, P., et al. 2014a, ApJ, 800, 38

Grillo, C., Gobat, R., Presotto, V., et al. 2014b, ApJ, 786, 11

Guennou, L., Adami, C., Durret, F., et al. 2014, A\&A, 516, A112

Guest, P. G. 1961, Numerical Methods of Curve Fitting (Cambridge: Cambridge Univ. Press)

Ishizaka, C. 1997, Ap\&SS, 254, 233

Jeltema, T. E., Canizares, C. R., Bautz, M. W., \& Buote, D. A. 2005, ApJ, 624, 606

Jones, C., \& Forman, W. 1999, ApJ, 511, 65

Jones, D. H., Saunders, W., Colless, M., et al. 2004, MNRAS, 355, 747

Lamareille, F., Contini, T., Le Borgne, J.-F., et al. 2006, A\&A, 448, 893

Ledermann, W. 1982, Handbook of Applicable Mathematics (New York: Wiley),

Vol. 6

Lemze, D., Postman, M., Genel, S., et al. 2013, ApJ, 776, 91

Lewis, I., Balogh, M., De Propris, R. et al. 2002, MNRAS, 334, 673

Ma, C.-J., Ebeling, E., Marshall, P., \& Schrabback, T. 2010, MNRAS, 406, 121

Mahajan, S., Mamon, G. A., Raychaudhury, S. 2011, MNRAS, 416, 2882

Mahajan, S., Raychaudhury, S., \& Pimbblet, K. A. 2012, MNRAS, 427, 1252

Maurogordato, S., Sauvageot, J. L., Bourdin, H., et al. 2011, A\&A, 525, A79

Melnick, J., \& Sargent, W. L. 1977, ApJ, 215, 401

Mercurio, A., Busarello, G., Merluzzi, P., et al. 2004, A\&A, 424, 79 (M04)
Mercurio, A., La Barbera, F., Haines, C. P., et al. 2008, MNRAS, 387, 1374

Moore, B., Diemand, J., \& Stadel, J. 2004, in Outskirts of Galaxy Clusters: Intense Life in the Suburbs, ed. A. Diaferio, IAU Colloq., \#195, 513

Moss, C., \& Dickens, R. J. 1977, 178, 701

Munari, E., Biviano, A., \& Mamon, G. 2014, A\&A, 566, A68

Mushotzky, R. F., \& Scharf, C. A. 1997, ApJ, 482, L13

Muzzin, A., van der Burg, R. F. J., McGee, S. L., et al. 2014, ApJ, 796, 65

Nonino, M., Dickinson, M., Rosati, P., et al. 2009, ApJS, 183, 244

Oemler, A. Jr., Dressler, A., Kelson, D., et al. 2009, ApJ, 693, 152

Owen, M. S., Ledlow, M. J., Keel, W. C., Wang, Q. D., \& Morrison, G. E. 2005, AJ, 129, 31

Owers, M. S., Randall, S. W., Nulsen, P. E., et al. 2011, ApJ, 728, 27

Pinkney, J., Roettiger, K., Burns, J. O., \& Bird, C. M. 1996, ApJS, 104, 1

Pisani, A. 1993, MNRAS, 265, 706

Pisani, A. 1996, MNRAS, 278, 697

Plionis, M., \& Basilakos, S. 2002, MNRAS, 329, L47

Poggianti, B. M., \& Barbaro, G. 1996, A\&A, 314, 379

Poggianti, B. M., \& Barbaro, G. 1997, A\&A, 325, 1025

Poggianti, B. M., Smail, I., Dressler, A., et al. 1999, ApJ, 518, 576

Poggianti, B. M., Bridges, T. J., Komiyama, Y., et al. 2004, ApJ, 601, 197

Poggianti, B. M., Aragón-Salamanca, A., Zaritsky, D., et al. 2009, ApJ, 693, 112

Porter, S. C., \& Raychaudhury, S. 2007, MNRAS, 375, 1409

Postman, M., Coe, M., Benítez, N., et al. 2012, ApJS, 199, 25

Presotto, V., Girardi, M., Nonino, M., et al. 2014, A\&A, 565, A126

Press, W. H., Teukolsky, S. A., Vetterling, W. T., \& Flannery, B. P. 1992, in

Numerical Recipes, 2nd edn. (Cambridge University Press)

Ramella, M., Biviano, A., Pisani, A., et al. 2007, A\&A, 470, 39

Rasmussen, J, Mulchaey, J. S., Bai, L., et al. 2012, ApJ, 757, 122

Rines, K., Geller, M. J., Kurtz, M. J., \& Diaferio, A., \& Kurtz, M. J. 2005, AJ, 130,1482

Rines, K., Geller, M. J., Diaferio, A., \& Kurtz, M. J. 2013, ApJ, 767, 15

Roettiger, K., Burns, J. O., \& Loken, C. 1996, ApJ, 473, 651

Rosati, P., Balestra, I., Grillo, C., et al. 2014, The Messenger, 158, 48

Rudick, C. S., Mihos, J. C., Frey, L. H., \& McBride, C. K. 2009, ApJ, 699, 1518

Schlegel, D. J., Finkbeiner, D. P., \& Davis, M. 1998, ApJ, 500, 525

Scodeggio, M., Franzetti, P., Garilli, B., et al. 2005, PASP, 117, 1284

Smith, G. P., Kneib, J.-P., Smail, I., et al. 2005, MNRAS, 359, 417

Sodré, L. Jr., Capelato, H. V., Steiner, J. E., \& Mazure, A. 1989, AJ, 97, 1279

Struck, C. 2006, in Atrophysics Update 2, ed. J. W. Mason (Heidelberg: Springer), 115

Tammann, G. A. 1972, A\&A, 21, 355

Treu, T., Ellis, R. S., Kneib, J.-P., et al. 2003, ApJ, 591, 53

Umetsu, K., Medezinski, E., Nonino, M., et al. 2012, ApJ, 755, 56

Vijayaraghavan, R., \& Ricker, P. M. 2013, MNRAS, 435, 2713

Wen, Z. L., \& Han, J. L. 2013, MNRAS, 436, 275

West, M. J., \& Bothun, G. D. 1990, ApJ, 350, 36

White, S. D. 1996 in Gravitational dynamics, Proc. 36th Herstmonceux conference, in honour of Professor D. Lynden-Bell's 60th birthday, Cambridge, UK, August 7-11, 1995 (Cambridge Univeristy Press), eds. O. Lahav, E. Terlevich, \& R. J. Terlevich, 121

Whitmore, B. C., Gilmore, D. M., \& Jones, C. 1993, ApJ, 407, 489

Young, A. H., Mroczkowski, T., \& Romero, C. 2014, ApJ, submitted [arXiv: 1411.0317]

Zabludoff, A. I., \& Franx, M. 1993, AJ, 106, 1314

Zhang, Y.-Y., Reiprich, T. H., Finoguenov, A., Hudson, D. S., \& Sarazin, C. L. 2009, ApJ, 699, 1178

Zitrin, A., Rosati, P., Nonino, M., et al. 2012, ApJ, 749, 97

1 Dipartimento di Fisica, Università degli Studi di Trieste, via Tiepolo 11, 34143 Trieste, Italy

e-mail: girardi@oats.inaf.it

2 INAF/Osservatorio Astronomico di Trieste, via G. B. Tiepolo 11, 34133, Trieste, Italy

3 INAF/Osservatorio Astronomico di Capodimonte, via Moiariello 1680131 Napoli, Italy

4 Dark Cosmology Centre, Niels Bohr Institute, University of Copenhagen, Juliane Maries Vej 30, 2100 Copenhagen, Denmark

5 Dipartimento di Fisica e Scienze della Terra, Università degli Studi di Ferrara, via Saragat 1, 44122 Ferrara, Italy

6 Department of Astronomy, Universidad de Concepcion, Casilla 160C, Concepcion, Chile

7 INAF/IASF-Milano, via Bassini 15, 20133 Milano, Italy

8 Korea Institute for Advanced Study, KIAS, 85 Hoegiro, 130-722 Dongdaemun-gu Seoul, Republic of Korea 
9 Department of Physics and Astronomy, The Johns Hopkins University, 3400 North Charles Street, Baltimore, MD 21218, USA

10 INAF/Osservatorio Astrofisico di Arcetri, Largo E. Fermi 5, 50125 Firenze, Italy

11 Space Telescope Science Institute, 3700 San Martin Drive, Baltimore, MD 21218, USA

12 Observatories of the Carnegie Institution of Washington, Pasadena, CA 91 101, USA

13 Dipartimento di Fisica, Università degli Studi di Milano, via Celoria 16, 20133 Milan, Italy

14 Institute of Astronomy and Astrophysics, Academia Sinica, PO Box 23-141, 10617 Taipei, Taiwan

15 California Institute of Technology, MC 249-17, Pasadena, CA 91125, USA

${ }^{16}$ Fundaciòn Galileo Galilei - INAF, Rambla José Ana Fernández Pérez 7, 38712 Breña Baja, La Palma, Spain
17 Instituto de Astrofísica de Canarias, C/Vía Láctea s/n, 38205 La Laguna, Tenerife, Spain

18 Universidad de La Laguna, Dpto. Astrofísica, 38206 La Laguna, Tenerife, Spain

19 University of Vienna, Department of Astrophysics, Türkenschanzstr. 17, 1180 Wien, Austria

20 INAF/Osservatorio Astronomico di Bologna, via Ranzani 1, 40127 Bologna, Italy

21 INFN, Sezione di Bologna, via Ranzani 1, 40127 Bologna, Italy

22 Jet Propulsion Laboratory, California Institute of Technology, 4800 Oak Grove Drive, Pasadena, CA 91109, USA

23 University Observatory Munich, Scheinerstrasse 1, 81679 München, Germany

24 Max-Planck-Institut für extraterrestrische Physik, Posfatch 1312, Giessenbachstr., 85741 Garching, Germany 